\title{
ASYMPTOTIC PROPERTIES OF DOUBLY ADAPTIVE BIASED COIN DESIGNS FOR MULTITREATMENT CLINICAL TRIALS
}

\author{
BY FEIFANG HU ${ }^{1}$ AND LI-XIN ZHANG ${ }^{2}$ \\ University of Virginia and Zhejiang University
}

\begin{abstract}
A general doubly adaptive biased coin design is proposed for the allocation of subjects to $K$ treatments in a clinical trial. This design follows the same spirit as Efron's biased coin design and applies to the cases where the desired allocation proportions are unknown, but estimated sequentially. Strong consistency, a law of the iterated logarithm and asymptotic normality of this design are obtained under some widely satisfied conditions. For two treatments, a new family of designs is proposed and shown to be less variable than both the randomized play-the-winner rule and the adaptive randomized design. Also the proposed design tends toward a randomization scheme (with a fixed target proportion) as the size of the experiment increases.
\end{abstract}

\section{Preliminaries.}

1.1. Brief history. Adaptive design is a sequential design in which design points or treatments are selected according to previously chosen design points or outcomes. Two main goals of adaptive designs are (i) to develop early stopping rules so that a trial can be terminated early with the possibility of reducing the overall number of patients on a randomized clinical trial and (ii) to maximize the total number of patients receiving the better treatment under certain restrictions.

The ideas of adaptive designs can be traced back to Thompson (1933) and Robbins (1952). Zelen (1969) proposed and studied the play-the-winner rule for comparing two treatments in clinical trials. In the play-the-winner rule, a success on one treatment results in the next patient's assignment to the same treatment, and a failure on one treatment results in the next patient's assignment to the opposite treatment. Since then, two main families of adaptive designs have been proposed: (1) response adaptive randomization, which is based on an optimal allocation target, where a specific criterion is optimized based on a population response model, and (2) design-driven response adaptive randomization, where

Received December 2001; revised March 2003.

${ }^{1}$ Supported in part by NSF Grant DMS-02-04232 and by Grant R-155-000-015-112 from the National University of Singapore.

${ }^{2}$ Supported by the National Natural Science Foundation of China and the National Natural Science Foundation of Zhejiang Province.

AMS 2000 subject classifications. 60F15, 62G10.

Key words and phrases. Adaptive randomized design, asymptotic normality, randomized playthe-winner rule, urn model. 
rules are established with intuitive motivation, but are not optimal in a formal sense [Rosenberger and Lachin (2002)]. Applications of adaptive designs in many different disciplines can be found in Flournoy and Rosenberger (1995).

1.2. Target allocation proportions. To achieve the two main goals of adaptive designs, optimal allocation proportions are usually determined according to some multiple-objective optimality criteria. This has been studied in Hayre (1979) and Jennison and Turnbull (2000), among others. Jennison and Turnbull (2000) describe a general procedure for determining optimal allocation.

Here we use binary response to illustrate different target allocation proportions. In comparing two treatments, 1 and 2, let the responses of treatment 1 follow a Bernoulli distribution with parameter $p_{1}$ and the responses of treatment 2 follow a Bernoulli with parameter $p_{2}$. Let $q_{1}=1-p_{1}$ and $q_{2}=1-p_{2}$. Suppose $n_{1}$ and $n_{2}$ are the numbers of patients assigned to treatments 1 and 2, respectively. Let $v=$ $n_{1} /\left(n_{1}+n_{2}\right)$ denote the allocation proportion to treatment 1 . For equal allocation, $v=1 / 2$. Neyman allocation, $v=\sqrt{p_{1} q_{1}} /\left(\sqrt{p_{1} q_{1}}+\sqrt{p_{2} q_{2}}\right)$, is proposed for minimizing the variance of $\hat{p}_{1}-\hat{p}_{2}$ [Melfi and Page (1998)]. Rosenberger, Stallard, Ivanova, Harper and Ricks (2001) propose the proportion $v=\sqrt{p_{1}} /\left(\sqrt{p_{1}}+\sqrt{p_{2}}\right)$ to maximize the expected number of successes $v n_{1} p_{1}+(1-v) n_{2} p_{2}$ with a fixed variance of $\hat{p}_{1}-\hat{p}_{2}$. Some other optimal proportions are considered in Ivanova and Rosenberger (2001).

1.3. Response-adaptive randomization procedures. Efron (1971) discussed the drawbacks of complete randomization and deterministic allocation for cases in which balance $(v=1 / 2)$ is desired. To overcome these drawbacks, Efron (1971) and Wei (1978) proposed biased coin designs that offer a compromise between complete randomization and deterministic allocation to reduce experimental bias and to increase the precision of inference about treatment difference. Smith (1984) and Wei, Smythe and Smith (1986) extended these designs to the multitreatment case when balance is desired or the desired allocation proportions are known.

In many sequential designs, balance is not desired and the desired allocation proportions may be unknown. For example, both $v=\sqrt{p_{1} q_{1}} /\left(\sqrt{p_{1} q_{1}}+\sqrt{p_{2} q_{2}}\right)$ and $v=\sqrt{p_{1}} /\left(\sqrt{p_{1}}+\sqrt{p_{2}}\right)$ depend on unknown parameters $p_{1}$ and $p_{2}$. To target unknown desired allocation proportions, Eisele (1994) and Eisele and Woodroofe (1995) introduced a doubly adaptive biased coin design for comparing two treatments. The design follows the same spirit of Efron's and Wei's designs, but depends on both the current proportion of subjects assigned to each treatment and the current estimate of the desired allocation proportion. Melfi, Page and Geraldes (2001) proposed an adaptive randomized design to target unknown proportions.

Different from the optimal allocation approach, a design-driven approach has been developed on an independent track. The basic idea is to use an intuitive rule (e.g., the play-the-winner rule) to change allocation probabilities sequentially. 
A well-studied family of adaptive designs is based on urn models, which include the randomized play-the-winner rule [Wei and Durham (1978)] as a special case. The main properties and some recent developments of urn models are reviewed in Rosenberger (2002).

1.4. Variability of randomization procedures. Much of the past literature on adaptive designs has focused on proposing new designs and evaluating properties of these designs. When a randomization procedure is used in a clinical study, the number of patients assigned to each treatment is a random variable. The variability of allocation can have a strong effect on power. This has been demonstrated by the simulation studies of Melfi and Page (1998) as well as Rosenberger, Stallard, Ivanova, Harper and Ricks (2001) and more recently theoretically by $\mathrm{Hu}$ and Rosenberger (2003), who show explicitly the relationship between the power of a test and the variability of the randomization procedure for a given allocation proportion. In that paper, they show that the average power of a randomization procedure is a decreasing function of the variability of the procedure. This allows us to directly evaluate different responseadaptive randomization procedures and different target allocations in terms of power and expected treatment failure rate without relying on simulation. So we can compare response-adaptive randomization procedures by studying their asymptotic distributions and especially their asymptotic variabilities. Therefore, it is important to propose new adaptive designs that have smaller variabilities and maintain certain degrees of randomness at the same time.

1.5. Main results and organization of the paper. Eisele and Woodroofe (1995) studied the asymptotic properties of doubly adaptive biased coin designs (for two treatments) under some very restrictive conditions on allocation functions. Indeed, as pointed out by Melfi, Page and Geraldes (2001): "the example given in their papers [Eisele $(1994,1995)]$ makes use of an allocation function which does not even satisfy these conditions!"

The main purposes of this paper are (i) to propose a new family of doubly adaptive biased coin designs for two treatments and show some advantages of the proposed designs, (ii) to generalize doubly adaptive biased coin designs for $K$-treatment comparisons and (iii) to study the asymptotic properties of generalized multitreatment doubly biased coin designs under some widely satisfied conditions. In this paper, we also show a law of the iterated logarithm of the doubly adaptive biased coin designs as well as the designs proposed by Wei (1978), Smith (1984) and Wei, Smythe and Smith (1986). By using martingale methods and the law of the iterated logarithm, we prove asymptotic normality of allocation proportions under some general conditions. It is shown that the allocation function of the example in Eisele $(1994,1995)$ satisfies the conditions here.

The paper is organized as follows. In Section 2, we propose a new family 
of doubly adaptive biased coin designs for two treatments, which is simple to implement and easy to understand for the practitioner. The new designs are compared with both the randomized play-the-winner rule [Wei and Durham (1978)] and the adaptive randomized design proposed by Melfi, Page and Geraldes (2001). We show that the new designs have smaller variabilities (force a small-sized experiment to desired allocation proportions) and tend toward the randomization scheme (see Remark 2.3) as the size of the experiment increases. Some examples are also discussed. General doubly biased coin designs for $K$ treatments are given in Section 3. In Section 4, we state some asymptotic properties, including strong consistency, law of the iterated logarithm and asymptotic normality, for both the allocation proportions and the estimates of the desired proportions. Based on these asymptotic properties, we show that the new design (for $K$ treatments) has the desired property that it converges toward the randomization scheme. We conclude our paper with some observations in Section 5. Technical proofs are given in the Appendix.

\section{Doubly adaptive biased coin design (DBCD) for two treatments.}

2.1. Allocation rule. For clinical trials with two treatments (1 and 2), let $\mathbf{X}_{m}=$ $\left(X_{m, 1}, X_{m, 2}\right)$ be the treatment assignment of the $m$ th patient. Here $\mathbf{X}_{m}=(1,0)$ indicates that the $m$ th patient is assigned to treatment 1 and $\mathbf{X}_{m}=(0,1)$ indicates that the $m$ th patient is assigned to treatment 2. Suppose $\left\{\left\{\boldsymbol{\xi}_{m, k}, m=1,2, \ldots\right\}\right.$, $k=1,2\}$ denote the responses, which are assumed to be two sequences (dependent) of i.i.d. random vectors in $\mathcal{R}^{d}$ (usually $d=1$ ), where $\boldsymbol{\xi}_{m, k}=\left(\xi_{m, k 1}, \ldots\right.$, $\left.\xi_{m, k d}\right)$ is the response of the $m$ th patient on the treatment $k, k=1,2$. In a real clinical trial, only $\boldsymbol{\xi}_{m, k}$ with $X_{m, k}=1(k=1,2)$ is observed.

Let $\mathbf{N}_{n}=\left(N_{n, 1}, N_{n, 2}\right)$, where $N_{n, k}=\sum_{j=1}^{n} X_{j, k}$ is the number of patients in treatment $k, k=1,2$, for the first $n$ patients and $N_{n, 1}+N_{n, 2}=n$. Let $\boldsymbol{\theta}_{1}$ and $\boldsymbol{\theta}_{2}$ be the corresponding parameters of treatments 1 and 2, respectively. Based on the first $n$ observations, let $\widehat{\boldsymbol{\theta}}_{n, 1}$ be the estimator of $\boldsymbol{\theta}_{1}$ and $\widehat{\boldsymbol{\theta}}_{n, 2}$ be the estimator $\boldsymbol{\theta}_{2}$. One goal of the allocation scheme is to have $N_{n, 1} / n \rightarrow v_{1}$ as $n \rightarrow \infty$, where $v_{1}=\rho\left(\boldsymbol{\theta}_{1}, \boldsymbol{\theta}_{2}\right)$ is the desired allocation proportion $\left[0 \leq \rho\left(\boldsymbol{\theta}_{1}, \boldsymbol{\theta}_{2}\right) \leq 1\right]$. Also let $g(x, y)$ be a function from $[1,0] \times[0,1]$ to $[0,1]$, which is called the allocation function.

Eisele (1994) and Eisele and Woodroofe (1995) propose the following doubly adaptive biased coin design (DBCD): (1) To start, allocate $n_{0} \geq 2$ patients to both treatments 1 and 2 , and (2) for the $(m+1)$ st stage $\left(m \geq 2 n_{0}\right)$, assign the $(m+1)$ st patient to treatment 1 with probability $g\left(N_{m, 1} / m, \widehat{\rho}_{m}\right)$, where $g$ is an allocation function and $\widehat{\rho}_{m}=\rho\left(\widehat{\boldsymbol{\theta}}_{m, 1}, \widehat{\boldsymbol{\theta}}_{m, 2}\right)$.

REMARK 2.1. Eisele and Woodroofe (1995) assume that both $\boldsymbol{\xi}_{m, 1}$ and $\boldsymbol{\xi}_{m, 2}$ are independent random variables from $d$-dimensional standard exponential 
families with $\boldsymbol{\theta}_{1}$ and $\boldsymbol{\theta}_{2}$ as their natural parameters, respectively. Here we consider general distributions of $\boldsymbol{\xi}_{m, 1}$ and $\boldsymbol{\xi}_{m, 2}$.

For simplicity of notation, we assume that both $\boldsymbol{\theta}_{1}$ and $\boldsymbol{\theta}_{2}$ are $d$-dimensional parameters and $\boldsymbol{\theta}_{1}=E \boldsymbol{\xi}_{1,1}$ and $\boldsymbol{\theta}_{2}=E \boldsymbol{\xi}_{1,2}$. Otherwise, for example, if there exist functions $\boldsymbol{f}_{k}$ such that $\boldsymbol{\theta}_{k}=E \boldsymbol{f}_{k}\left(\boldsymbol{\xi}_{1, k}\right), k=1,2$, then we can use transformations $\boldsymbol{f}_{k}$ and treat these as the responses. This includes the case of Eisele and Woodroofe (1995). For MLEs, moment estimators and estimators from estimate equations, this condition is usually satisfied (asymptotically) after some modifications. This is demonstrated in Example 1.

Based on the above discussion, we have $\boldsymbol{\theta}_{1}=\left(\theta_{11}, \ldots, \theta_{1 d}\right)$ and $\boldsymbol{\theta}_{2}=$ $\left(\theta_{21}, \ldots, \theta_{2 d}\right)$. We will use sample means to estimate the parameters $\boldsymbol{\theta}_{1}$ and $\boldsymbol{\theta}_{2}$, that is,

$$
\widehat{\boldsymbol{\theta}}_{m, k}=\frac{\sum_{j=1}^{m} X_{j, k} \boldsymbol{\xi}_{j, k}}{N_{m, k}}, \quad k=1,2 .
$$

2.2. Conditions on the allocation function $g$ and asymptotic results. The properties of the DBCD depend heavily on the allocation function $g$. To show the asymptotic properties of the DBCD, Eisele and Woodroofe (1995) assume the following conditions of the allocation function $g$ and the desired proportion function $\rho$ :

(i) $g$ is jointly continuous;

(ii) $g(x, x)=x$ for all $x \in[0,1]$; $(0,1)$;

(iii) $g(x, y)$ is strictly decreasing in $x$ and strictly increasing in $y$ on $(0,1) \times$

(iv) $g(x, y)$ has bounded partial derivatives in both $x$ and $y$ and $\partial g(x, y) /$ $\left.\partial x\right|_{x=v_{1}, y=v_{1}} \neq 0$;

(v) there are positive constants $C$ and $\gamma$ for which

$$
\frac{1}{\rho}+\frac{1}{1-\rho} \leq C\left(\left\|E\left(\xi_{1,1}\right)\right\|^{\gamma}+\left\|E\left(\xi_{1,2}\right)\right\|^{\gamma}\right) ;
$$

(vi) $\rho$ is a continuous function and it is twice continuously differentiable in a small neighborhood of $\left(\boldsymbol{\theta}_{1}, \boldsymbol{\theta}_{2}\right)$.

Conditions (iv) and (v) are very restrictive, and it is usually difficult to check these two conditions. In fact, Melfi, Page and Geraldes (2001) pointed out that the function $g(x, y)=[1-(1 / y-1) x]^{+}$[cf. Eisele $\left.(1994,1995)\right]$ does not satisfy condition (iv). This is because $\partial g /\left.\partial x\right|_{y=x}=1-1 / x$ is not bounded. Condition (v) is usually not satisfied for $\rho \rightarrow 0$ or $\rho \rightarrow 1$. In this paper, we will avoid these two conditions.

Suppose $v_{1}=\rho\left(\boldsymbol{\theta}_{1}, \boldsymbol{\theta}_{2}\right)$ is the desired allocation proportion. We introduce the following condition: 
(vii) there exists $\delta>0$ such that $g(x, y)$ satisfies

$$
\begin{aligned}
g(x, y)= & g\left(v_{1}, v_{1}\right)+\left.\left(x-v_{1}\right) \frac{\partial g}{\partial x}\right|_{\left(v_{1}, v_{1}\right)}+\left.\left(y-v_{1}\right) \frac{\partial g}{\partial y}\right|_{\left(v_{1}, v_{1}\right)} \\
& +o\left(\left|x-v_{1}\right|^{1+\delta}\right)+o\left(\left|y-v_{1}\right|^{1+\delta}\right)
\end{aligned}
$$

as $(x, y) \rightarrow\left(v_{1}, v_{1}\right)$.

Now let

$$
\lambda=\left.\frac{\partial g}{\partial x}\right|_{\left(v_{1}, v_{1}\right)}, \quad \gamma=\left.\frac{\partial g}{\partial y}\right|_{\left(v_{1}, v_{1}\right)}
$$

and

$$
\nabla(\rho)=\left(\frac{\partial \rho}{\partial \theta_{11}}, \ldots, \frac{\partial \rho}{\partial \theta_{1 d}}, \frac{\partial \rho}{\partial \theta_{21}}, \ldots, \frac{\partial \rho}{\partial \theta_{2 d}}\right)^{\prime}
$$

Also let

$$
\sigma_{3}^{2}=\left.\left(\left.\nabla(\rho)\right|_{\Theta}\right)^{\prime} \mathbf{V} \nabla(\rho)\right|_{\Theta} \quad \text { and } \quad \sigma_{1}^{2}=v_{1}\left(1-v_{1}\right),
$$

where $\boldsymbol{\Theta}=\left(\boldsymbol{\theta}_{1}, \boldsymbol{\theta}_{2}\right)$ and

$$
\mathbf{V}=\operatorname{diag}\left(\frac{\operatorname{Var}\left(\boldsymbol{\xi}_{1,1}\right)}{v_{1}}, \frac{\operatorname{Var}\left(\boldsymbol{\xi}_{1,2}\right)}{1-v_{1}}\right)
$$

THEOREM 2.1. If (i)-(iii), (vi) and (vii) are satisfied and $E\left\|\boldsymbol{\xi}_{1, k}\right\|^{2+\varepsilon}<\infty$, $k=1,2$, for some $\varepsilon>0$, then

$$
\begin{gathered}
\frac{N_{n, 1}}{n}-v_{1}=O\left(\sqrt{\frac{\log \log n}{n}}\right) \quad \text { a.s. }, \\
n^{-1 / 2}\left(N_{[n t], 1}-[n t] v_{1},[n t] \widehat{\rho}_{[n t]}-[n t] v_{1}\right) \\
\stackrel{D}{\rightarrow}\left(\sigma_{1} t^{\lambda} \int_{0}^{t} \frac{d W_{t}}{x^{\lambda}}+\gamma \sigma_{3} t^{\lambda} \int_{0}^{t} \frac{B_{x}}{x^{1+\lambda}} d x, \sigma_{3} B_{t}\right)
\end{gathered}
$$

and

$$
n^{1 / 2}\left(N_{n, 1} / n-v_{1}, \widehat{\rho}_{n}-v_{1}\right) \stackrel{\mathcal{D}}{\rightarrow} N(\mathbf{0}, \mathbf{\Sigma}),
$$

where $W_{t}$ and $B_{t}$ are two independent standard Brownian motions and

$$
\boldsymbol{\Sigma}=\left(\begin{array}{cc}
\sigma_{1}^{2} /(1-2 \lambda)+2 \gamma^{2} \sigma_{3}^{2} /((1-\lambda)(1-2 \lambda)) & \gamma \sigma_{3}^{2} /(1-\lambda) \\
\gamma \sigma_{3}^{2} /(1-\lambda) & \sigma_{3}^{2}
\end{array}\right)
$$


Results (2.3) and (2.2) agree with Theorems 5 and 6 of Eisele and Woodroofe (1995), respectively. Result (2.1) is new. Only conditions (i)-(iii), (vi) and (vii) are used in this theorem. Condition (vii) is a local condition and is not difficult to check, while condition (iv) of Eisele and Woodroofe (1995) is a global condition. The $g(x, y)=[1-(1 / y-1) x]^{+}$satisfies condition (vii) here. Condition (v) is not assumed in this paper.

REMARK 2.2. Theorem 2.1 is a special case of Theorems 4.1-4.3 (see Remark 4.2). It is easy to check the conditions of Theorems 4.1-4.3 from the conditions of Theorem 2.1. The condition $\lambda<1 / 2$ is used in Section 4. From condition (iii) of Theorem 2.1, we have $\lambda<0$, so $\lambda<1 / 2$ is satisfied. For example, $\lambda=1-1 / v_{1}<0$ for $g(x, y)=[1-(1 / y-1) x]^{+}$. It is true in other examples of this paper.

2.3. A family of allocation functions and its properties. From Theorem 2.1, the allocation function $g(x, y)$ plays an important role. If we choose $g(x, y)=$ $1-x$ and $v_{1}=v_{2}=1 / 2$, we get the adaptive biased coin design proposed by Wei (1978). If $g(x, y)=p(2 x-1)$, then we obtain the adaptive biased coin designs studied by Wei (1978) and Smith (1984). Generally, we consider the following family of allocation functions.

A family of allocation functions. Define the allocation function

$$
\begin{aligned}
& g^{(\alpha)}(0, y)=1, \quad g^{(\alpha)}(1, y)=0, \\
& g^{(\alpha)}(x, y)=\frac{y(y / x)^{\alpha}}{y(y / x)^{\alpha}+(1-y)((1-y) /(1-x))^{\alpha}},
\end{aligned}
$$

where $\alpha \geq 0$. It is easy to see that, if $x>y$, then $g(x, y)<y$. This will force the allocation proportion to the target proportion, which follows the same spirit as Efron's biased coin design (1971). Also DBCDs based on this family of allocation functions are easy to implement because the value is easy to calculate. The asymptotic properties of this family of designs can be obtained from Theorem 2.1 with $\lambda=-\alpha<1 / 2, \gamma=1+\alpha$. Now we use the following two examples to illustrate the properties of this family of allocation functions.

EXAMPLE 1 (Normal responses). This is the example studied in Section 8 of Eisele and Woodroofe (1995). Suppose

$$
Y_{1}, Y_{2}, \ldots \sim N\left(\mu, \sigma^{2}\right) \text { and } Z_{1}, Z_{2}, \ldots \sim N\left(v, \tau^{2}\right)
$$

where $\mu, v, \sigma^{2}$ and $\tau^{2}$ are four unknown parameters. To test $\mu=v$, the desired proportion is

$$
v_{1}=\frac{\sigma}{\sigma+\tau}
$$


which is not a direct function of $E\left(Y_{1}\right)$ and $E\left(Z_{1}\right)$. Let $\boldsymbol{\xi}_{m, 1}=\left(Y_{m}^{2}, Y_{m}\right)$ and $\xi_{m, 2}=\left(Z_{m}^{2}, Z_{m}\right)$. In this case, $\theta_{11}=\mathrm{E} Y_{1}^{2}, \theta_{12}=\mathrm{E} Y_{1}=\mu, \theta_{21}=\mathrm{E} Z_{1}^{2}, \theta_{22}=$ $\mathrm{E} Z_{1}=v$. Then

$$
v_{1}=\rho(\boldsymbol{\Theta})=\frac{\sigma}{\sigma+\tau}=\frac{\sqrt{\theta_{11}-\theta_{12}^{2}}}{\sqrt{\theta_{11}-\theta_{12}^{2}}+\sqrt{\theta_{21}-\theta_{22}^{2}}},
$$

and the estimates of $\sigma^{2}$ and $\tau^{2}$ are

$$
\widehat{\sigma}_{m}^{2}=\widehat{\theta}_{m, 11}-\left(\widehat{\theta}_{m, 12}\right)^{2}=\frac{1}{N_{m, 1}}\left(\sum_{j=1}^{m} X_{j, 1}\left(Y_{j}-\bar{Y}_{m}\right)^{2}\right)
$$

and

$$
\widehat{\tau}_{m}^{2}=\widehat{\theta}_{m, 21}-\left(\widehat{\theta}_{m, 22}\right)^{2}=\frac{1}{N_{m, 1}}\left(\sum_{j=1}^{m} X_{j, 2}\left(Z_{j}-\bar{Z}_{m}\right)^{2}\right),
$$

where

$$
\bar{Y}_{m}=\frac{\sum_{j=1}^{m} X_{j, 1} Y_{j}}{N_{m, 1}} \quad \text { and } \quad \bar{Z}_{m}=\frac{\sum_{j=1}^{m} X_{j, 2} Z_{j}}{N_{m, 2}} .
$$

Obviously, the function $\rho(\mathbf{y})$ is continuous in $\left\{\mathbf{y}: y_{11}>y_{12}^{2}, y_{21}>y_{22}^{2}\right\}$ and is twice differentiable at $\Theta$. Also,

$$
\left.\nabla(\rho)\right|_{\Theta}=\left(\frac{\tau}{2 \sigma(\sigma+\tau)^{2}},-\frac{2 \mu \tau}{2 \sigma(\sigma+\tau)^{2}},-\frac{\sigma}{2 \tau(\sigma+\tau)^{2}}, \frac{2 \nu \sigma}{2 \tau(\sigma+\tau)^{2}}\right)^{\prime} .
$$

Note that $(1,-2 \mu) \operatorname{Var}\left\{\left(Y^{2}, Y\right)\right\}(1,-2 \mu)^{\prime}=\operatorname{Var}\left\{(Y-\mu)^{2}\right\}=2 \sigma^{4}$ and $(1,-2 v) \times$ $\operatorname{Var}\left\{\left(Z^{2}, Z\right)\right\}(1,-2 v)^{\prime}=\operatorname{Var}\left\{(Z-v)^{2}\right\}=2 \tau^{4}$. We have

$$
\sigma_{3}^{2}=\frac{\tau^{2} 2 \sigma^{4}}{4 \sigma^{2}(\sigma+\tau)^{4}} \frac{\sigma+\tau}{\sigma}+\frac{\sigma^{2} 2 \tau^{4}}{4 \tau^{2}(\sigma+\tau)^{4}} \frac{\sigma+\tau}{\tau}=\frac{\tau \sigma}{2(\sigma+\tau)^{2}} .
$$

Therefore, if the allocation function satisfies the conditions of Theorem 2.1, then

$$
\frac{N_{n, 1}}{n}-v_{1}=O\left(\sqrt{\frac{\log \log n}{n}}\right) \quad \text { a.s. }
$$

and

$$
n^{-1 / 2}\left(N_{n, 1}-n v_{1}\right) \stackrel{\mathcal{D}}{\rightarrow} N\left(0,\left[\frac{1}{1-2 \lambda}+\frac{\gamma^{2}}{(1-\lambda)(1-2 \lambda)}\right] \frac{\sigma \tau}{(\sigma+\tau)^{2}}\right) .
$$

If the allocation function $g^{(\alpha)}$ of (2.4) is used, the conditions of Theorem 2.1 are satisfied and

$$
n^{-1 / 2}\left(N_{n, 1}-n v_{1}\right) \stackrel{\mathcal{D}}{\rightarrow} N\left(0, \frac{2+\alpha}{(1+2 \alpha)} \frac{\sigma \tau}{(\sigma+\tau)^{2}}\right) .
$$


The asymptotic variance is a monotone decreasing function of $\alpha$, taking values from $2 \sigma \tau /(\sigma+\tau)^{2}$ to $\sigma \tau /\left[2(\sigma+\tau)^{2}\right]$ as $\alpha$ changes from 0 to $\infty$.

When $\alpha=0, g^{(0)}(x, y)=y$, which does not depend on $x$. The DBCD becomes the adaptive randomized design proposed by Melfi, Page and Geraldes (2001). In this case, the asymptotic variance of $n^{-1 / 2} N_{n, 1}$ is $2 \sigma \tau /(\sigma+\tau)^{2}$. One disadvantage of the adaptive randomized design is that, for small experiments, the allocation could be far from the target proportion. This can be seen in the special case with a fixed $\rho=1 / 2$ [Efron (1971)]. The DBCD with $g^{(\alpha)}(\alpha>0)$ always has smaller asymptotic variance than the adaptive randomized design. If $\alpha=1$, the variance of DBCD is $\sigma \tau /(\sigma+\tau)^{2}$, which is half of the variance of the adaptive randomized design. Therefore, we can force a small-sized experiment to the target proportion by choosing $\alpha$ of the proposed designs.

EXAMPLE 2 (Binary responses). Suppose the response of a patient on each treatment is dichotomous (i.e., success or failure). Let $p_{1}=\mathrm{P}($ success $\mid$ treatment 1$)$, $p_{2}=\mathrm{P}($ success $\mid$ treatment 2$), q_{1}=1-p_{1}$ and $q_{2}=1-p_{2}$. Assume that $0<p_{1}<1$ and $0<p_{2}<1$. The randomized play-the-winner (RPW) rule [Wei and Durham (1978)] is often used for ethical reasons. If we use the RPW rule to assign the patients, then

$$
\frac{N_{n, 1}}{n} \rightarrow v_{1}=: \frac{q_{2}}{q_{1}+q_{2}} \quad \text { a.s. } \quad \text { and } \quad \sqrt{n}\left(\frac{N_{n, 1}}{n}-v_{1}\right) \stackrel{\mathcal{D}}{\rightarrow} N\left(0, \sigma_{\mathrm{RPW}}^{2}\right),
$$

whenever $p_{1}+p_{2}<3 / 2$ (or $\left.q_{1}+q_{2}>1 / 2\right)$, where

$$
\sigma_{\mathrm{RPW}}^{2}=\frac{q_{1} q_{2}\left[5-2\left(q_{1}+q_{2}\right)\right]}{\left[2\left(q_{1}+q_{2}\right)-1\right]\left(q_{1}+q_{2}\right)^{2}}
$$

[cf. Smythe and Rosenberger (1995)]. When $p_{1}+p_{2}>3 / 2$, the limiting distributions of the proportions are unknown for the RPW rule [cf. Matthews and Rosenberger (1997)].

Now we use the doubly biased coin design to target the same allocation proportions $q_{2} /\left(q_{1}+q_{2}\right)$. Here, $\rho\left(p_{1}, p_{2}\right)=\left(1-p_{2}\right) /\left[\left(1-p_{1}\right)+\left(1-p_{2}\right)\right]$. Let $g(x, y)$ be an allocation function that satisfies the conditions of Theorem 2.1. The design is defined as follows. At the first stage, $n_{0}$ patients are assigned to each treatment. After $m \geq 2 n_{0}$ patients are assigned, we let $S_{m, k}$ be the number of successes of all the $N_{m, k}$ patients on the treatment $k$ in the first $m$ assignments, $k=1,2$. We let $\widehat{p}_{m, k}=\left(S_{m, k}+1 / 2\right) /\left(N_{m, k}+1\right)$ be the sample estimator of $p_{k}$ and write $\widehat{q}_{m, k}=1-\widehat{p}_{m, k}, k=1,2$. At the $(m+1)$ st stage, the $(m+1)$ st patient is assigned to treatment 1 with probability $g\left(N_{m, 1} / m, \widehat{\rho}_{m}\right)$, and to treatment 2 with probability $1-g\left(N_{m, 1} / m, \widehat{\rho}_{m}\right)$, where $\widehat{\rho}_{m}=\widehat{q}_{m, 2} /\left(\widehat{q}_{m, 1}+\widehat{q}_{m, 2}\right)$ is the sample estimate of $v_{1}=q_{2} /\left(q_{1}+q_{2}\right)$. Then $\left.\nabla(\rho)\right|_{\left(p_{1}, p_{2}\right)}=\left(q_{2} /\left(q_{1}+q_{2}\right)^{2},-q_{1} /\left(q_{1}+\right.\right.$ $\left.q_{2}\right)^{2}$ ),

$$
\frac{N_{n, 1}}{n}-v_{1}=O\left(\sqrt{\frac{\log \log n}{n}}\right) \quad \text { a.s. } \quad \text { and } \quad n^{1 / 2}\left(\frac{N_{n, 1}}{n}-v_{1}\right) \stackrel{\mathcal{D}}{\rightarrow} N\left(0, \sigma_{\mathrm{DBC}}^{2}\right)
$$


whenever $\lambda<1 / 2$, where

$$
\sigma_{\mathrm{DBC}}^{2}=\frac{q_{1} q_{2}}{(1-2 \lambda)\left(q_{1}+q_{2}\right)^{2}}+\frac{2 \gamma^{2}}{(1-\lambda)(1-2 \lambda)} \frac{q_{1} q_{2}\left(p_{1}+p_{2}\right)}{\left(q_{1}+q_{2}\right)^{3}} .
$$

If $g(x, \rho)$ is chosen from $(2.4)$, then

$$
\begin{aligned}
\sigma_{\mathrm{DBC}}^{2} & =\sigma_{\alpha}^{2}=\frac{q_{1} q_{2}}{(1+2 \alpha)\left(q_{1}+q_{2}\right)^{2}}+\frac{2(1+\alpha)}{(1+2 \alpha)} \frac{q_{1} q_{2}\left(p_{1}+p_{2}\right)}{\left(q_{1}+q_{2}\right)^{3}} \\
& =\frac{q_{1} q_{2}\left(p_{1}+p_{2}\right)}{\left(q_{1}+q_{2}\right)^{3}}+\frac{2 q_{1} q_{2}}{(1+2 \alpha)\left(q_{1}+q_{2}\right)^{3}} .
\end{aligned}
$$

We shall note that the asymptotic normality holds for all $0<p_{1}<1$ and $0<$ $p_{2}<1$. It is easily seen that $\sigma_{\alpha}^{2}$ is a strictly decreasing function of $\alpha \geq 0$. Also, $\sigma_{\alpha}^{2}<\sigma_{\mathrm{RPW}}^{2}$ for all $\alpha>1$. Furthermore, if $q_{1}+q_{2}$ is near $1 / 2$, then $\sigma_{\alpha}$ is much smaller than $\sigma_{\mathrm{RPW}}$. Therefore, the doubly adaptive biased coin design provides a more stable allocation rule.

REMARK 2.3. From the above two examples (for any fixed $\alpha$ ), we find that, as the size $m$ of the experiment approaches $\infty, N_{m, 1} / m \rightarrow v_{1}, \widehat{\rho}_{m} \rightarrow v_{1}$ and $g\left(N_{m, 1} / m, \widehat{\rho}_{m}\right) \rightarrow v_{1}$ almost surely. Therefore, the $(m+1)$ st patient is assigned to treatment 1 with probability $g\left(N_{m, 1} / m, \widehat{\rho}_{m}\right) \rightarrow v_{1}$ as $m \rightarrow \infty$. Therefore, the new designs tend to the randomization scheme with target $v_{1}$ as $m$ increases.

3. The general model and the assumptions. We now consider general $K$-treatment clinical trials. Suppose the patients are randomized sequentially and respond immediately. In general, after $m$ patients are assigned and the responses observed, the $(m+1)$ st patient is assigned to treatment $k$, with a probability $\left\{p_{m, k}\right\}, k=1, \ldots, K$. The probabilities $\left\{p_{m, k}\right\}$ may depend on both the treatments assigned and the responses observed of the previous $m$ patients. Let $\mathbf{X}_{m}=\left(X_{m, 1}, \ldots, X_{m, K}\right)$ be the result of the $m$ th assignment; that is, if the $m$ th patient is assigned to treatment $k$, then the $k$ th component $X_{m, k}$ of $\mathbf{X}_{m}$ is 1 and other components are 0 . We let $\left\{\left\{\boldsymbol{\xi}_{m, k}, m=1,2, \ldots\right\}, k=1, \ldots, K\right\}$ denote the responses, which are assumed to be $K$ sequences of i.i.d. random vectors in $\mathcal{R}^{d}$, where $\boldsymbol{\xi}_{m, k}=\left(\xi_{m, k 1}, \ldots, \xi_{m, k d}\right)$ is the response of the $m$ th patient on treatment $k$, $k=1, \ldots, K$. We also write $\boldsymbol{\xi}_{m}=\left(\boldsymbol{\xi}_{m, 1}, \ldots, \boldsymbol{\xi}_{m, K}\right)$. Then the probabilities $p_{m, k}$, $k=1, \ldots, K$, are $K$ functions of $\mathbf{X}_{j}$ and $\xi_{j}, j=1, \ldots, m$. Since a treatment may have several dependent responses (e.g., cure, negative effect, side effect, etc.), we use a vector to denote a response of a patient on a treatment. Actually, in the clinical trial, $\boldsymbol{\xi}_{m, k}$ appears only when the $m$ th patient is assigned to treatment $k$, that is, when $X_{m, k}=1$. But we should assume that all the responses $\left\{\boldsymbol{\xi}_{m, k}\right\}$ are there, and only the nonzero element of $X_{m, k} \boldsymbol{\xi}_{m, k}$ is observed in the trial.

Let $\mathbf{N}_{n}=\left(N_{n, 1}, \ldots, N_{n, K}\right)$, where $N_{n, k}=\sum_{j=1}^{n} X_{j, k}$ is the number of patients assigned to the treatment $k$ in the first $m$ stages, $k=1, \ldots, K$. Suppose the 
desired allocation proportion of patients assigned to each treatment is a function of some unknown parameters of the response $\left\{\boldsymbol{\xi}_{n}\right\}$ [see Melfi and Page (1998) and Rosenberger, Stallard, Ivanova, Harper and Ricks (2001) for some special cases]. One goal of the allocation scheme is to have $\mathbf{N}_{n} / n \rightarrow \mathbf{v}=\boldsymbol{\rho}(\boldsymbol{\Theta})$ as $n \rightarrow \infty$, where $\rho(\mathbf{z})=\left(\rho_{1}(\mathbf{z}), \ldots, \rho_{K}(\mathbf{z})\right): \mathcal{R}^{d_{1} \times K} \rightarrow(0,1)^{K}$ is a vector-valued function satisfying $\boldsymbol{\rho}(\mathbf{z}) \mathbf{1}^{\prime}=1, \boldsymbol{\Theta}=\left(\boldsymbol{\theta}_{1}, \ldots, \boldsymbol{\theta}_{K}\right)$ is a vector in $\mathcal{R}^{d_{1} \times K}$ and $\boldsymbol{\theta}_{k}=$ $\left(\theta_{k 1}, \ldots, \theta_{k d_{1}}\right)$ is an unknown parameter of the distribution of $\boldsymbol{\xi}_{1, k}, k=1, \ldots, K$. For simplicity of notation, we assume that $d=d_{1}$ and $\boldsymbol{\theta}_{k}=\mathrm{E} \boldsymbol{\xi}_{1, k}, k=1, \ldots, K$. Otherwise, for example, if there exist functions $\mathbf{f}_{k}$ such that $\boldsymbol{\theta}_{k}=\mathrm{Ef}_{k}\left(\boldsymbol{\xi}_{1, k}\right)$, $k=1, \ldots, K$, we can use the transforms $\mathbf{f}_{k}\left(\boldsymbol{\xi}_{1, k}\right), k=1, \ldots, K$, of the responses as the responses themselves. (This has been demonstrated in Example 1, where $d=1$, but $d_{1}=2$. Transformations are used to make $d=2$.)

Choose a $\boldsymbol{\Theta}_{0}=\left(\boldsymbol{\theta}_{0,1}, \ldots, \boldsymbol{\theta}_{0, K}\right) \in \mathcal{R}^{d \times K}$ as the first estimate of $\boldsymbol{\Theta}$. If $m$ patients are assigned and the responses are observed, we use the sample means to estimate the parameters $\boldsymbol{\theta}_{k}, k=1, \ldots, K$, that is,

$$
\widehat{\boldsymbol{\theta}}_{m, k}=\frac{\sum_{j=1}^{m} X_{j, k} \boldsymbol{\xi}_{j, k}+\boldsymbol{\theta}_{0, k}}{N_{m, k}+1}, \quad k=1, \ldots, K,
$$

and write

$$
\widehat{\boldsymbol{\Theta}}_{m, k}=\left(\widehat{\boldsymbol{\theta}}_{m, 1}, \ldots, \widehat{\boldsymbol{\theta}}_{m, K}\right) .
$$

Here, 1 is added in the denominator to avoid the case of $0 / 0$, and $\boldsymbol{\theta}_{0, k}$ is added in the numerator to estimate $\boldsymbol{\theta}_{k}$ when no patient is assigned to treatment $k$, $k=1, \ldots, K$. Usually, $\boldsymbol{\Theta}_{0}$ is chosen to avoid $\rho_{k}\left(\widehat{\boldsymbol{\Theta}}_{m}\right)=0, k=1, \ldots, K$. In practice, $\boldsymbol{\Theta}_{0}$ is a guessed value of $\boldsymbol{\Theta}$ or an estimator of $\boldsymbol{\Theta}$ from other early trials.

Now, we consider the following general adaptive design.

General doubly adaptive biased coin design. Let $\mathbf{g}(\mathbf{x}, \mathbf{y})=\left(g_{1}(\mathbf{x}, \mathbf{y}), \ldots\right.$, $\left.g_{K}(\mathbf{x}, \mathbf{y})\right):[0,1]^{K} \times[0,1]^{K} \rightarrow[0,1]^{K}$ be the allocation rule with $\mathbf{g}(\mathbf{x}, \mathbf{y}) \mathbf{1}^{\prime}=1$. The first patient is allocated to each treatment with the same probability $1 / K$. Let $\widehat{\boldsymbol{\Theta}}_{m}$ be estimated as in (3.1) from the first $m$ observations, $m=1,2, \ldots$ Then the $(m+1)$ st patient is assigned to treatment $k$ with probability $p_{m, k}=$ $g_{k}\left(\mathbf{N}_{m} / m, \widehat{\boldsymbol{\rho}}_{m}\right), k=1, \ldots, K$, where

$$
\widehat{\boldsymbol{\rho}}_{m}=\rho\left(\widehat{\boldsymbol{\Theta}}_{m}\right)
$$

is the sample estimate of $\mathbf{v}=\left(v_{1}, \ldots, v_{K}\right)=\rho(\boldsymbol{\Theta})$ based on the responses observed from the first $m$ patients.

This design has the desirable property that it offers a compromise between randomization and balance. To obtain the asymptotic properties, we require the following assumptions. In this paper, we assume that $0<v_{k}<1, k=1, \ldots, K$. The following first group of conditions concerns the response $\left\{\xi_{n}=\left(\xi_{n, 1}, \ldots, \xi_{n, K}\right)\right\}$ and related parameters $\boldsymbol{\Theta}=\left(\boldsymbol{\theta}_{1}, \ldots, \boldsymbol{\theta}_{K}\right)=\left(\theta_{11}, \ldots, \theta_{1 d}, \ldots, \theta_{k 1}, \ldots, \theta_{k d}\right)$. 
Condition A. Assume that the response sequence $\left\{\boldsymbol{\xi}_{n}=\left(\boldsymbol{\xi}_{n, 1}, \ldots, \boldsymbol{\xi}_{n, K}\right)\right\}$, $n=1,2, \ldots$, is i.i.d. random vectors and $\boldsymbol{\Theta}=\mathrm{E} \boldsymbol{\xi}_{n}$ as before. Also:

(A1) $\mathrm{E}\left\|\boldsymbol{\xi}_{1, k}\right\|<\infty, k=1, \ldots, K$.

(A2) For some $\varepsilon>0$, E $\left\|\xi_{1, k}\right\|^{2+\varepsilon}<\infty, k=1, \ldots, K$.

The second group of conditions concerns the allocation rule $\mathbf{g}(\mathbf{x}, \mathbf{y})=\left(g_{1}(\mathbf{x}, \mathbf{y})\right.$, $\left.\ldots, g_{K}(\mathbf{x}, \mathbf{y})\right)$.

Condition B. The function of allocation rule $\mathbf{g}(\mathbf{x}, \mathbf{y})$ satisfies the following conditions:

(B1) $\mathbf{g}(\mathbf{v}, \mathbf{v})=\mathbf{v}$ and $\mathbf{g}(\mathbf{x}, \mathbf{y})-\mathbf{g}(\mathbf{x}, \mathbf{v}) \rightarrow \mathbf{0}$ as $\mathbf{y} \rightarrow \mathbf{v}$ along $\mathbf{y} \mathbf{1}^{\prime}=1$ uniformly in $\mathbf{x}$ with $\mathbf{x} \mathbf{1}^{\prime}=1$. The latter can be implied by the condition that the function $\mathbf{g}(\mathbf{x}, \mathbf{y})$ is continuous on $\left\{(\mathbf{x}, \mathbf{v}): \mathbf{x} \mathbf{1}^{\prime}=1\right\}$.

(B2) There exists a constant $0 \leq \lambda_{0}<1$ such that, for each $k=1, \ldots, K$,

$$
\frac{g_{k}(\mathbf{x}, \mathbf{v})-g_{k}(\mathbf{v}, \mathbf{v})}{x_{k}-v_{k}} \leq \lambda_{0} \quad \text { for all } \mathbf{x} \text { with } x_{k}>v_{k}, \mathbf{x} \mathbf{1}^{\prime}=1 .
$$

(B3) For any $0<\delta<1 / K$ and each $k=1, \ldots, K$, there exists a constant $c_{\delta}>0$ such that

$$
\begin{aligned}
& \left.g_{k}(\mathbf{x}, \mathbf{y})\right|_{x_{k}=0} \geq c_{\delta} \quad \text { for all } \mathbf{x}, \mathbf{y} \\
& \text { with } \mathbf{x} \mathbf{1}^{\prime}=1, \mathbf{y} \mathbf{1}^{\prime}=1, \mathbf{y} \in[\delta, 1)^{K} \text {, } \\
& \liminf _{x_{k} \rightarrow 0^{+}} \frac{g_{k}(\mathbf{x}, \mathbf{y})}{\min \left\{x_{1}, \ldots, x_{K}\right\}} \geq c_{\delta} \quad \text { uniformly in } \mathbf{x}, \mathbf{y} \\
& \text { with } \mathbf{x} \mathbf{1}^{\prime}=1, \mathbf{y} \mathbf{1}^{\prime}=1, \mathbf{y} \in[\delta, 1)^{K} .
\end{aligned}
$$

(B4) There exists $\delta>0$ for which the function $\rho(\mathbf{x}, \mathbf{y})$ satisfies

$$
\begin{aligned}
\mathbf{g}(\mathbf{x}, \mathbf{y})= & \mathbf{g}(\mathbf{v}, \mathbf{v})+\left.\sum_{k=1}^{K}\left(x_{k}-v_{k}\right) \frac{\partial \mathbf{g}}{\partial x_{k}}\right|_{(\mathbf{v}, \mathbf{v})}+\left.\sum_{k=1}^{K}\left(y_{k}-v_{k}\right) \frac{\partial \mathbf{g}}{\partial u_{k}}\right|_{(\mathbf{v}, \mathbf{v})} \\
& +o\left(\|\mathbf{x}-\mathbf{v}\|^{1+\delta}\right)+o\left(\|\mathbf{y}-\mathbf{v}\|^{1+\delta}\right) \quad \text { as }(\mathbf{x}, \mathbf{y}) \rightarrow(\mathbf{v}, \mathbf{v}) .
\end{aligned}
$$

The third group of conditions concerns the proportion function $\rho(\mathbf{z})$.

CONDITION C. The proportion function $\mathbf{z}=\left(\mathbf{z}_{1}, \ldots, \mathbf{z}_{d}\right)=\left(z_{11}, \ldots, z_{1 d}, \ldots\right.$, $\left.z_{K 1}, \ldots, z_{K d}\right) \rightarrow \boldsymbol{\rho}(\mathbf{z}): \mathcal{R}^{d \times K} \rightarrow(0,1)^{K}$ satisfies the following conditions:

(C1) $\rho(\boldsymbol{\Theta})=\mathbf{v}$ and $\rho(\mathbf{z})$ is a continuous function.

(C2) There exists $\delta>0$ for which

$$
\boldsymbol{\rho}(\mathbf{z})=\boldsymbol{\rho}(\boldsymbol{\Theta})+\left.\sum_{k=1}^{K} \sum_{j=1}^{d}\left(z_{k j}-\theta_{k j}\right) \frac{\partial \boldsymbol{\rho}}{\partial z_{k j}}\right|_{\boldsymbol{\Theta}}+o\left(\|\mathbf{z}-\boldsymbol{\Theta}\|^{1+\delta}\right) \quad \text { as } \mathbf{z} \rightarrow \boldsymbol{\Theta} .
$$


For a function $\mathbf{h}(\mathbf{u}, \mathbf{w}): \mathcal{R}^{L} \times \mathcal{R}^{M} \rightarrow \mathcal{R}^{K}$, we denote by $\nabla_{\mathbf{u}}(\mathbf{h})$ and $\nabla_{\mathbf{w}}(\mathbf{h})$ the gradient matrices related to the vectors $\mathbf{u}$ and $\mathbf{w}$, respectively, that is,

$$
\nabla_{\mathbf{u}}(\mathbf{h})=\left(\frac{\partial h_{k}}{\partial u_{i}} ; i=1, \ldots, L, k=1, \ldots, K\right)_{L \times K}
$$

and

$$
\nabla_{\mathbf{w}}(\mathbf{h})=\left(\frac{\partial h_{k}}{\partial w_{j}} ; j=1, \ldots, M, k=1, \ldots, K\right)_{M \times K} .
$$

If conditions (B4) and (C2) are satisfied, then

$$
\begin{aligned}
\mathbf{g}(\mathbf{x}, \boldsymbol{\rho}(\mathbf{z}))= & \mathbf{g}(\mathbf{v}, \mathbf{v})+(\mathbf{x}-\mathbf{v}) \mathbf{H}+\left.(\mathbf{z}-\boldsymbol{\Theta}) \nabla(\boldsymbol{\rho})\right|_{\boldsymbol{\Theta}} \mathbf{E} \\
& +o\left(\|\mathbf{x}-\mathbf{v}\|^{1+\delta}\right)+o\left(\|\mathbf{z}-\boldsymbol{\Theta}\|^{1+\delta}\right)
\end{aligned}
$$

as $(\mathbf{x}, \mathbf{z}) \rightarrow(\mathbf{v}, \boldsymbol{\Theta})$, where

$$
\mathbf{H}=\left.\nabla_{\mathbf{x}}(\mathbf{g})\right|_{(\mathbf{v}, \mathbf{v})} \quad \text { and } \quad \mathbf{E}=\left.\nabla_{\rho}(\mathbf{g})\right|_{(\mathbf{v}, \mathbf{v})}
$$

are two $K \times K$ matrices, and

$$
\left.\nabla_{\mathbf{y}}(\mathbf{f})\right|_{(\mathbf{v}, \boldsymbol{\Theta})}=\left.\left.\nabla(\boldsymbol{\rho})\right|_{\boldsymbol{\Theta}} \nabla_{\boldsymbol{\rho}}(\mathbf{g})\right|_{(\mathbf{v}, \mathbf{v})}=\left.\nabla(\boldsymbol{\rho})\right|_{\boldsymbol{\Theta}} \mathbf{E}
$$

is a $(d \cdot K) \times K$ matrix. Obviously, $\mathbf{H} \mathbf{1}^{\prime}=\mathbf{E} \mathbf{1}^{\prime}=\mathbf{0}^{\prime}$ since $\mathbf{g}(\mathbf{x}, \mathbf{y}) \mathbf{1}^{\prime}=1$. Therefore, $\mathbf{H}$ has an eigenvalue $\lambda_{1}=0$ and has the following Jordan decomposition:

$$
\mathbf{T}^{-1} \mathbf{H T}=\operatorname{diag}\left[0, \mathbf{J}_{2}, \ldots, \mathbf{J}_{s}\right],
$$

where $\mathbf{J}_{s}$ is a $v_{t} \times v_{t}$ matrix, given by

$$
\mathbf{J}_{t}=\left(\begin{array}{ccccc}
\lambda_{t} & 1 & 0 & \cdots & 0 \\
0 & \lambda_{t} & 1 & \cdots & 0 \\
0 & 0 & \lambda_{t} & \cdots & 0 \\
\vdots & \vdots & \vdots & \ddots & \vdots \\
0 & 0 & 0 & \cdots & \lambda_{t}
\end{array}\right) .
$$

We may select the matrix $\mathbf{T}$ so that its first column is $\mathbf{1}^{\prime}$. Let $\lambda=\max \left\{\operatorname{Re}\left(\lambda_{2}\right), \ldots\right.$, $\left.\operatorname{Re}\left(\lambda_{s}\right)\right\}$ and $v=\max _{j}\left\{v_{j}: \operatorname{Re}\left(\lambda_{j}\right)=\lambda\right\}$.

Further, if condition (B2) is also satisfied, then due to an argument similar to that made in Section 3 of Smith (1984) or in the proof of Lemma 3.2 of Wei, Smythe and Smith (1986),

$$
\mathbf{T}^{-1} \mathbf{H T}=\operatorname{diag}[0, \lambda, \ldots, \lambda] \quad \text { and } \quad \mathbf{H}=\lambda \mathbf{H}_{0}=\lambda\left(\mathbf{I}-\mathbf{1}^{\prime} \mathbf{u}\right),
$$

where $\mathbf{H}_{0}=\mathbf{T} \operatorname{diag}[0,1, \ldots, 1] \mathbf{T}^{-1}=\mathbf{I}-\mathbf{1}^{\prime} \mathbf{u}, \mathbf{u}$ is the first row of $\mathbf{T}^{-1}$.

We will use conditions (A1), (B1)-(B3) and (C1) to establish the strong consistency, and conditions (A2), (B4) and (C2) to establish asymptotic normality. We now make some remarks on the assumptions. 
REMARK 3.1. If $\mathbf{g}(\mathbf{x}, \mathbf{y})$ and $\rho(\mathbf{z})$ are twice differentiable at points $(\mathbf{v}, \mathbf{v})$ and $\boldsymbol{\Theta}$, respectively, or the second partial derivatives of them are bounded in a neighborhood of the points $(\mathbf{v}, \mathbf{v})$ and $\boldsymbol{\Theta}$, respectively, then conditions (B4) and (C2) are satisfied with $\delta=3 / 4$.

REMARK 3.2. Condition (B2) is satisfied with $\lambda_{0}=0$ if we assume that the $(m+1)$ st patient is assigned to treatment $k$ with a probability less than $v_{k}$ whenever $N_{m, k} / m>v_{k}$. In this case, the biased coin design analyzed by Smith (1984) and Wei, Smythe and Smith (1986) is a special case of our general adaptive design. Their $\mathbf{g}(\mathbf{x}, \boldsymbol{\rho})$ does not depend on $\boldsymbol{\rho}$. By symmetry, condition (B2) can replaced by:

(B2') There exists a constant $0 \leq \lambda_{0}<1$ such that, for each $k=1, \ldots, K$,

$$
\frac{g_{k}(\mathbf{x}, \mathbf{v})-g_{k}(\mathbf{v}, \mathbf{v})}{x_{k}-v_{k}} \leq \lambda_{0} \quad \text { for all } \mathbf{x} \text { with } x_{k}<v_{k}, \mathbf{x} \mathbf{1}^{\prime}=1 .
$$

Or a general one:

(B2 $\left.{ }^{\prime \prime}\right)$ There exist two constants $0 \leq \lambda_{0}<1, c_{0} \neq 0$ and an invertible real matrix $\mathbf{S}=\left(\mathbf{s}_{1}^{\prime}, \ldots, \mathbf{s}_{K}^{\prime}\right)$ such that $\mathbf{S} \mathbf{1}^{\prime}=c_{0} \mathbf{1}^{\prime}$ and, for each $k=1, \ldots, K$,

$$
\frac{(\mathbf{g}(\mathbf{x}, \mathbf{v})-\mathbf{g}(\mathbf{v}, \mathbf{v})) \mathbf{s}_{k}^{\prime}}{(\mathbf{x}-\mathbf{v}) \mathbf{s}_{k}^{\prime}} \leq \lambda_{0} \quad \text { for all } \mathbf{x} \text { with }(\mathbf{x}-\mathbf{v}) \mathbf{s}_{k}^{\prime}>0, \mathbf{x} \mathbf{1}^{\prime}=1 .
$$

In these cases, (3.4) remains true. In practice, we can choose $\mathbf{g}$ such that one of condition (B2), (B2') and (B2') is satisfied.

REMARK 3.3. Condition (B3) is easily understood. At the stage $(m+1)$, if all estimated proportions $\widehat{\rho}_{m, j}, j=1, \ldots, K$, are not very small, but the sample proportion $N_{m, k} / m$ is very small, then the probability of the assignment of the $(m+1)$ st patient to the treatment $k$ should not be too small to avoid large experimental bias. This condition can simply be replaced by the condition that

$$
N_{n, k} \rightarrow \infty \quad \text { a.s., } k=1, \ldots, K .
$$

If $\mathbf{g}(\mathbf{x}, \mathbf{y})=\mathbf{g}(\mathbf{x})$ is only a function of $\mathbf{x}$, then condition (B3) is not needed, since this condition or (3.5) is only used for ensuring the consistency of $\widehat{\boldsymbol{\Theta}}_{n}$ (cf. Lemmas A.4 and A.5). Also, if condition (B2') is satisfied at any point ( $\mathbf{v}, \mathbf{v})$ and $g_{k}(\mathbf{v}, \mathbf{v})=v_{k}$, then (B3) is obviously satisfied. Therefore, conditions (ii) and (iii) of Eisele $(1994,1995)$ or Eisele and Woodroofe (1995) imply this condition.

REMARK 3.4. Our conditions on the allocation rule are weaker than those used by Smith (1984). Conditions (B1)-(B3) are weaker than conditions (i)-(iii) in Eisele (1994, 1995) or Eisele and Woodroofe (1995). Their (iv) is a global condition; our (B4) is a local one instead. Also, condition (C2) is weaker than their (vi). Any condition of the form of their condition (v) is not assumed in this paper. It is usually difficult to verify their (v) in applications. 
4. Asymptotic properties. In this section we state the main asymptotic theorems.

THEOREM 4.1 (Strong consistency). If conditions (A1), (B1), (B2"), (B3) and $(\mathrm{C} 1)$ are satisfied, then $\mathbf{N}_{n} / n \rightarrow \mathbf{v}$ and $\widehat{\boldsymbol{\rho}}_{n} \rightarrow \mathbf{v}$ a.s.

THEOREM 4.2 (Rates of consistency). Suppose $\mathbf{N}_{n} / n \rightarrow \mathbf{v}$ a.s. If conditions (A2), (B4) and (C2) are satisfied and $\lambda<1$, then for any $\kappa>(1 / 2) \vee \lambda$,

$$
n^{-\kappa}\left(\mathbf{N}_{n}-n \mathbf{v}\right) \rightarrow 0 \quad \text { a.s. } \quad \text { and } \quad \widehat{\boldsymbol{\rho}}_{n}-\mathbf{v}=O\left(\sqrt{\frac{\log \log n}{n}}\right) \quad \text { a.s. }
$$

Furthermore, if $\lambda<1 / 2$, then

$$
\mathbf{N}_{n}-n \mathbf{v}=O(\sqrt{n \log \log n}) \quad \text { a.s. }
$$

THEOREM 4.3 (Asymptotic normality). Suppose $\mathbf{N}_{n} / n \rightarrow \mathbf{v}$ a.s. and conditions (A2), (B4) and (C2) are satisfied. Let

$$
\mathbf{V}_{k}=\operatorname{Var}\left(\xi_{1, k}\right)=\left(\operatorname{Cov}\left[\xi_{1, k i}, \xi_{1, k j}\right] ; i, j=1, \ldots, d\right), \quad k=1, \ldots, K
$$

$$
\mathbf{V}=\operatorname{diag}\left(\frac{1}{v_{1}} \mathbf{V}_{1}, \ldots, \frac{1}{v_{K}} \mathbf{V}_{K}\right)
$$

$$
\boldsymbol{\Sigma}_{3}=\left.\left(\left.\nabla(\boldsymbol{\rho})\right|_{\Theta}\right)^{\prime} \mathbf{V} \nabla(\boldsymbol{\rho})\right|_{\boldsymbol{\Theta}}=\left.\sum_{k=1}^{K} \frac{1}{v_{k}}\left(\left.\nabla_{\mathbf{y}_{k}}(\boldsymbol{\rho})\right|_{\boldsymbol{\Theta}}\right)^{\prime} \mathbf{V}_{k} \nabla_{\mathbf{y}_{k}}(\boldsymbol{\rho})\right|_{\boldsymbol{\Theta}},
$$

(4.4) $\quad \boldsymbol{\Sigma}_{1}=\operatorname{diag}(\mathbf{v})-\mathbf{v}^{\prime} \mathbf{v}, \quad \boldsymbol{\Sigma}_{2}=\mathbf{E}^{\prime} \boldsymbol{\Sigma}_{3} \mathbf{E}$,

and let $\mathbf{W}_{t}$ and $\mathbf{B}_{t}$ be two independent standard $K$-dimensional Brownian motions. If $\lambda<1 / 2$, then

$$
n^{-1 / 2}\left(\mathbf{N}_{[n t]}-[n t] \mathbf{v},[n t] \widehat{\boldsymbol{\rho}}_{[n t]}-[n t] \mathbf{v}\right) \stackrel{\mathscr{D}}{\rightarrow}\left(\mathbf{G}_{t}, \mathbf{B}_{t} \boldsymbol{\Sigma}_{3}^{1 / 2}\right)
$$

in the space $\mathscr{D}_{[0,1]}$ with the Skorohod topology, where the Gaussian process

$$
\begin{aligned}
\mathbf{G}_{t} & =\int_{0}^{t}\left(d \mathbf{W}_{x}\right) \Sigma_{1}^{1 / 2}\left(\frac{t}{x}\right)^{\mathbf{H}}+\int_{0}^{t} \frac{\mathbf{B}_{x} \boldsymbol{\Sigma}_{2}^{1 / 2}}{x}\left(\frac{t}{x}\right)^{\mathbf{H}} d x \\
& =\int_{0}^{t}\left(d \mathbf{W}_{x}\right) \Sigma_{1}^{1 / 2}\left(\frac{t}{x}\right)^{\mathbf{H}}+\int_{0}^{t}\left(d \mathbf{B}_{x}\right) \Sigma_{2}^{1 / 2}\left[\int_{x}^{t} \frac{1}{y}\left(\frac{t}{y}\right)^{\mathbf{H}} d y\right]
\end{aligned}
$$

is the solution of the equation

$$
d \mathbf{G}_{t}=\left(d \mathbf{W}_{t}\right) \Sigma_{1}^{1 / 2}+\frac{\mathbf{B}_{t} \boldsymbol{\Sigma}_{2}^{1 / 2}}{t} d t+\frac{\mathbf{G}_{t}}{t} \mathbf{H} d t, \quad \mathbf{G}_{0}=\mathbf{0},
$$


and $a^{\mathbf{H}}$ is defined to be

$$
a^{\mathbf{H}}=e^{\mathbf{H} \ln a}=\sum_{j=0}^{\infty} \frac{(\ln a)^{j}}{j !} \mathbf{H}^{k} .
$$

In particular,

$$
n^{1 / 2}\left(\mathbf{N}_{n} / n-\mathbf{v}, \widehat{\boldsymbol{\rho}}_{n}-\mathbf{v}\right) \stackrel{\mathcal{D}}{\rightarrow} N(0, \mathbf{\Lambda})
$$

where

$$
\boldsymbol{\Lambda}=\left(\begin{array}{cc}
\boldsymbol{\Lambda}_{11} & \boldsymbol{\Lambda}_{12} \\
\boldsymbol{\Lambda}_{21} & \boldsymbol{\Sigma}_{3}
\end{array}\right)
$$

and

$$
\begin{aligned}
\boldsymbol{\Lambda}_{11}= & \int_{0}^{1}\left(\frac{1}{x}\right)^{\mathbf{H}^{\prime}} \boldsymbol{\Sigma}_{1}\left(\frac{1}{x}\right)^{\mathbf{H}} d x \\
& +\int_{0}^{1} d x\left[\int_{x}^{1} \frac{1}{y}\left(\frac{1}{y}\right)^{\mathbf{H}} d y\right]^{\prime} \boldsymbol{\Sigma}_{2}\left[\int_{x}^{1} \frac{1}{y}\left(\frac{1}{y}\right)^{\mathbf{H}} d y\right],
\end{aligned}
$$

$$
\boldsymbol{\Lambda}_{12}^{\prime}=\boldsymbol{\Lambda}_{21}=\int_{0}^{1} d x \boldsymbol{\Sigma}_{3} \mathbf{E}\left[\int_{x}^{1} \frac{1}{y}\left(\frac{1}{y}\right)^{\mathbf{H}} d y\right]=\boldsymbol{\Sigma}_{3} \mathbf{E}(\mathbf{I}-\mathbf{H})^{-1} .
$$

REMARK 4.1. If condition (B2) or (B2") is satisfied, then by (3.4),

$$
a^{\mathbf{H}}=\sum_{j=0}^{\infty} \frac{(\lambda \log a)^{j}}{j !} \mathbf{H}_{0}=a^{\lambda} \mathbf{H}_{0}=a^{\lambda}\left(\mathbf{I}-\mathbf{1}^{\prime} \mathbf{u}\right) .
$$

Also, $\boldsymbol{\Sigma}_{1} \mathbf{1}^{\prime}=\mathbf{0}$ and $\mathbf{E} \mathbf{1}^{\prime}=\mathbf{0}$. Therefore, $\mathbf{H}_{0}^{\prime} \boldsymbol{\Sigma}_{1} \mathbf{H}_{0}=\boldsymbol{\Sigma}_{1}, \mathbf{H}_{0}^{\prime} \boldsymbol{\Sigma}_{2} \mathbf{H}_{0}=\boldsymbol{\Sigma}_{2}$ and $\mathbf{E H}_{0}=\mathbf{E}$. It follows that

$$
\begin{aligned}
& \boldsymbol{\Lambda}_{11}=\frac{\mathbf{H}_{0}^{\prime} \boldsymbol{\Sigma}_{1} \mathbf{H}_{0}}{1-2 \lambda}+\frac{2 \mathbf{H}_{0}^{\prime} \boldsymbol{\Sigma}_{2} \mathbf{H}_{0}}{(1-\lambda)(1-2 \lambda)}=\frac{\boldsymbol{\Sigma}_{1}}{1-2 \lambda}+\frac{2 \boldsymbol{\Sigma}_{2}}{(1-\lambda)(1-2 \lambda)}, \\
& \boldsymbol{\Lambda}_{12}^{\prime}=\boldsymbol{\Lambda}_{21}=\frac{1}{1-\lambda} \boldsymbol{\Sigma}_{3} \mathbf{E} \mathbf{H}_{0}=\frac{1}{1-\lambda} \boldsymbol{\Sigma}_{3} \mathbf{E}
\end{aligned}
$$

and

$$
\begin{aligned}
\mathbf{G}_{t} & =t^{\lambda} \int_{0}^{t} \frac{\left(d \mathbf{W}_{x}\right) \boldsymbol{\Sigma}_{1}^{1 / 2} \mathbf{H}_{0}}{x^{\lambda}}+t^{\lambda} \int_{0}^{t} \frac{\mathbf{B}_{x} \boldsymbol{\Sigma}_{2}^{1 / 2}}{x^{\lambda+1}} d x \\
& =t^{\lambda} \int_{0}^{t} \frac{\left(d \mathbf{W}_{x}\right) \boldsymbol{\Sigma}_{1}^{1 / 2}}{x^{\lambda}}+t^{\lambda} \int_{0}^{t} \frac{\mathbf{B}_{x} \boldsymbol{\Sigma}_{2}^{1 / 2}}{x^{\lambda+1}} d x .
\end{aligned}
$$

The first part of $\mathbf{G}_{t}$ is a Gaussian process with covariance function $t^{\lambda} s^{1-\lambda} \boldsymbol{\Sigma}_{1} /(1-$ $2 \lambda$ ), which agrees with (3.1) of Smith (1984). If the desired allocation proportions are known, then the second part of $\mathbf{G}_{t}$ does not appear since $\mathbf{E}=\mathbf{0}$ and $\boldsymbol{\Sigma}_{2}=\mathbf{0}$. 
REMARK 4.2. For Theorem $2.1(K=2)$, we have

$$
\boldsymbol{\Sigma}_{1}=\sigma_{1}^{2}\left(\begin{array}{rr}
1 & -1 \\
-1 & 1
\end{array}\right), \quad \boldsymbol{\Sigma}_{2}=\gamma^{2} \sigma_{3}^{2}\left(\begin{array}{rr}
1 & -1 \\
-1 & 1
\end{array}\right), \quad \boldsymbol{\Sigma}_{3}=\sigma_{3}^{2}\left(\begin{array}{rr}
1 & -1 \\
-1 & 1
\end{array}\right) .
$$

REMARK 4.3. Theorem 4.1 shows that the allocation tends toward the randomization scheme with the desired probabilities as the size of the experiment increases. Theorem 4.2 provides the law of the iterated logarithm of the designs. This theorem also applies to the adaptive bias coin designs [Wei (1978)] and the designs in Smith (1984) and Wei, Smythe and Smith (1986). The general variancecovariance formula in Theorem 4.3 is very important because it can be used when comparing the design with other sequential designs. The results of the above three theorems can be easily extended to the estimate of the parameter $\Theta$.

Now we give an example for the multitreatment clinical trial.

EXAmple 3. Consider the $K$-treatment adaptive design, $K \geq 2$. Suppose the responses of patients on each treatment are also dichotomous (i.e., success or failure). Let $p_{k}=\mathrm{P}($ success $\mid$ treatment $k)$ and $q_{k}=1-p_{k}, k=1, \ldots, K$. Suppose $0<p_{k}<1, k=1, \ldots, K$. As an extension of the RPW rule, Wei (1979) proposed a generalized Pólya's urn (GPU) design in that it also assigns more patients to better treatments and allows delayed response by the patient. By using his design, the limiting proportions of patients assigned are

$$
v_{1}=\frac{1 / q_{1}}{\sum_{j=1}^{K} 1 / q_{j}}, \ldots, v_{K}=\frac{1 / q_{K}}{\sum_{j=1}^{K} 1 / q_{j}} .
$$

However, the limiting distribution of the sample proportions $\mathbf{N}_{n}$ strongly depends on the eigenvalues of the generating matrix of the model:

$$
\mathbf{M}=\left(\begin{array}{cccc}
p_{1} & q_{1} /(K-1) & \cdots & q_{1} /(K-1) \\
q_{2} /(K-1) & p_{2} & \cdots & q_{1} /(K-1) \\
\cdots & \cdots & \cdots & \cdots \\
q_{K} /(K-1) & q_{K} /(K-1) & \cdots & p_{K}
\end{array}\right) .
$$

Let $\tau=\max \left\{\operatorname{Re}\left(\tau_{2}\right), \ldots, \operatorname{Re}\left(\tau_{K}\right)\right\}$, where $\tau_{1}=1, \tau_{2}, \ldots, \tau_{K}$ are all the eigenvalues of $\mathbf{M}$. Then the asymptotic normality of $\mathbf{N}_{n}$ holds only when $\tau \leq 1 / 2$ and the variances are very large when $\tau$ equals or is near 1/2 [cf. Smythe (1996) and Bai and $\mathrm{Hu}$ (1999)]. However, when $K \geq 3$, the expression for $\tau$ becomes very complex; it is very hard (even impossible in more general cases) to verify the condition $\tau \leq 1 / 2$.

Now we use the doubly biased coin to assign the patients. We can choose the allocation function $\mathbf{g}(\mathbf{x}, \mathbf{y})$ to be

$$
g_{k}(\mathbf{x}, \mathbf{y})=\frac{\left\{y_{k}\left(y_{k} / x_{k}\right)^{\alpha}\right\} \wedge L}{\sum_{j=1}^{K}\left\{y_{j}\left(y_{j} / x_{j}\right)^{\alpha}\right\} \wedge L}, \quad k=1, \ldots, K,
$$


where $\alpha \geq 0$ and $L>1$ are constants. Here the function $g$ depends on the constant $L$ for technical reasons, but we can choose large $L$ to reduce its influence. For this function,

$$
\mathbf{H}=-\alpha\left(\begin{array}{cccc}
1-v_{1} & -v_{2} & \cdots & -v_{K} \\
-v_{1} & 1-v_{2} & \cdots & -v_{K} \\
\cdots & \cdots & \cdots & \cdots \\
-v_{1} & -v_{2} & \cdots & 1-v_{K}
\end{array}\right)=:-\alpha \mathbf{H}_{0} \quad \text { and } \quad \mathbf{E}=(1+\alpha) \mathbf{H}_{0}
$$

where $\mathbf{H}_{0}=\mathbf{I}-\mathbf{1}^{\prime} \mathbf{v}$. Obviously, $\mathbf{g}(\mathbf{x}, \mathbf{y})$ satisfies conditions (B1) and (B4). Also,

$$
g_{k}(\mathbf{x}, \mathbf{y}) \geq \frac{\left\{y_{k}\left(y_{k} / x_{k}\right)^{\alpha}\right\} \wedge L}{K L} .
$$

Therefore, (B3) is satisfied. For verifying condition (B2), we let $f_{k}\left(x_{k}\right)=$ $\left\{v_{k}\left(v_{k} / x_{k}\right)^{\alpha}\right\} \wedge L, T(\mathbf{x})=\sum_{k=1}^{k} f_{k}\left(x_{k}\right)$. Obviously, $f_{k}\left(x_{k}\right)<v_{k}$ if $x_{k}>v_{k}, k=$ $1, \ldots, K$, and

$$
\min \left\{\sum_{k=1}^{K} v_{k}\left(\frac{v_{k}}{x_{k}}\right)^{\alpha}: \sum_{j=1}^{K} x_{k}=1,0<x_{k}<1, k=1, \ldots, K\right\}=\sum_{k=1}^{K} v_{k}=1 .
$$

It follows that $T(\mathbf{x}) \geq 1$. Therefore,

$$
g_{k}(\mathbf{x}, \mathbf{v})-v_{k}=\frac{f_{k}\left(x_{k}\right)-v_{k}}{T(\mathbf{x})}+v_{k}\left(\frac{1}{T(\mathbf{x})}-1\right) \leq 0 \quad \text { if } x_{k}>v_{k} .
$$

Condition (B2) is satisfied. Furthermore, since $\mathbf{H}_{0} \mathbf{1}^{\prime}=\mathbf{0}, \nabla(\boldsymbol{\rho}) \mathbf{1}^{\prime}=\mathbf{0}$ and $\mathbf{v} \mathbf{1}^{\prime}=1$, we have

$\boldsymbol{\Sigma}_{2}=(1+\alpha)^{2} \mathbf{H}_{0}^{\prime} \boldsymbol{\Sigma}_{3} \mathbf{H}_{0}=(1+\alpha)^{2} \boldsymbol{\Sigma}_{3} \quad$ and $\quad \boldsymbol{\Sigma}_{3} \mathbf{E}=(1+\alpha) \boldsymbol{\Sigma}_{3} \mathbf{H}_{0}=(1+\alpha) \boldsymbol{\Sigma}_{3}$.

So, if $\boldsymbol{\rho}(\mathbf{z})$ is chosen to satisfy conditions (C1) and (C2), then by Theorems 4.1-4.3 and Remark 3.1,

$$
\begin{array}{ll}
\frac{\mathbf{N}_{n}}{n}-\mathbf{v}=O\left(\sqrt{\frac{\log \log n}{n}}\right) \quad \text { a.s., } \\
\widehat{\boldsymbol{\rho}}_{n}-\mathbf{v}=O\left(\sqrt{\frac{\log \log n}{n}}\right) \quad \text { a.s. }
\end{array}
$$

and

$$
n^{1 / 2}\left(\mathbf{N}_{n} / n-\mathbf{v}, \widehat{\boldsymbol{\rho}}_{n}-\mathbf{v}\right) \stackrel{\mathcal{D}}{\rightarrow} N(\mathbf{0}, \boldsymbol{\Lambda})
$$

where

$$
\boldsymbol{\Lambda}=\left(\begin{array}{cc}
(1 /(1+2 \alpha)) \boldsymbol{\Sigma}_{1}+(2(1+\alpha) /(1+2 \alpha)) \boldsymbol{\Sigma}_{3} & \boldsymbol{\Sigma}_{3} \\
\boldsymbol{\Sigma}_{3} & \boldsymbol{\Sigma}_{3}
\end{array}\right)
$$


Also, $\boldsymbol{\Lambda}_{11} \rightarrow \boldsymbol{\Sigma}_{3}$ as $\alpha \rightarrow+\infty$. If the desired allocation proportions are the same as those in Wei's design, then the proportion function $\rho(\mathbf{z})$ is

$$
\rho_{k}\left(z_{1}, \ldots, z_{K}\right)=\frac{1 /\left(1-z_{k}\right)}{\sum_{j=1}^{K} 1 /\left(1-z_{j}\right)}, \quad k=1, \ldots, K,
$$

and the estimate of $\mathbf{p}=\left(p_{1}, \ldots, p_{k}\right)$ is

$$
\left(\frac{S_{m, 1}+1 / 2}{N_{m, 1}+1}, \ldots, \frac{S_{m, K}+1 / 2}{N_{m, K}+1}\right),
$$

where $S_{m, k}$ is the number of successes of all the $N_{m, k}$ patients on treatment $k$ in the first $m$ stages. In this case,

$$
\begin{aligned}
\boldsymbol{\Sigma}_{3} & =\left(\left.\nabla(\boldsymbol{\rho})\right|_{\mathbf{p}}\right)^{\prime} \operatorname{diag}\left(p_{1} q_{1} / v_{1}, \ldots, p_{K} q_{K} / v_{K}\right)\left(\left.\nabla(\boldsymbol{\rho})\right|_{\mathbf{p}}\right) \\
& =\sum_{j=1}^{K} \frac{p_{j} v_{j}}{q_{j}}\left(\mathbf{v}-\mathbf{e}_{j}\right)^{\prime}\left(\mathbf{v}-\mathbf{e}_{j}\right),
\end{aligned}
$$

where $\mathbf{e}_{j}$ is the vector of which the $j$ th component is 1 and the others are 0 .

Also, if the desired allocation proportions are $\left(\sqrt{p_{1} q_{1}}, \ldots, \sqrt{p_{K} q_{K}}\right) /$ $\sum_{j=1}^{K} \sqrt{p_{j} q_{j}}$, then we can choose

$$
\boldsymbol{\rho}(\mathbf{z})=\left(\sqrt{z_{1}\left(1-z_{1}\right)}, \ldots, \sqrt{z_{K}\left(1-z_{K}\right)}\right) / \sum_{j=1}^{K} \sqrt{z_{j}\left(1-z_{j}\right)} .
$$

5. Conclusions. The asymptotic properties of general multiarm doubly adaptive biased coin designs under widely satisfied conditions are important in their own right. But the main contributions of this paper are in proposing the family of doubly adaptive biased coin designs for $K=2$ and showing the two important properties of the designs: (i) they have smaller variabilities than the randomized play-the-winner rule and the adaptive randomized design, and (ii) they tend to the randomization scheme as the number of patients increases.

For the proposed family of designs, we have also demonstrated, in Examples 1 and 2, that the asymptotic variance of the proposed design is a decreasing function of $\alpha$. When $\alpha=0$, it leads to the adaptive randomized design. When $\alpha=\infty$, the variance of the design is minimized, but the design is then completely deterministic. The practitioners can choose $\alpha$ to balance the degree of randomness and the variation in applications.

As noted in Remark 2.1, Eisele and Woodroofe (1995) assume that the responses are from standard exponential distributions. In this paper, we only assume that the responses have $2+\varepsilon$ moment $(\varepsilon>0)$. So the results in this paper can be widely applied. The randomized play-the-winner rule is usually applied to binary responses, while the doubly adaptive biased coined design can be applied to both discrete responses and continuous responses. 
Another advantage of the doubly adaptive biased coin design is that it can target any given allocation proportion, while the urn model [Rosenberger (2002)] can only target a specific allocation proportion. In Example 2, the randomized playthe-winner rule can only target the specific $v_{1}=q_{2} /\left(q_{1}+q_{2}\right)$. But the doubly adaptive biased coin design can target the Neyman allocation and others.

For clinical trials with delayed responses, we can update the urn when the response becomes available for urn models. This was first suggested by Wei (1988) for the randomized play-the-winner rule. Some asymptotic properties of the urn model with delayed responses are studied in Bai, Hu and Rosenberger (2003). For doubly adaptive biased coin designs, delayed responses will affect the estimators of unknown parameters. Their asymptotic properties with delayed responses are unknown, but we leave this topic for future research. Another topic of future research is applying the doubly adaptive biased coin design to survival responses.

From the theorems of Sections 2 and 4, the asymptotic properties of the doubly adaptive biased coin design depend on both the allocation function $g$ and the target function $\rho$. Selection of $\rho$ can be found in Jennison and Turnbull (2000). In applications, the allocation function $g^{(\alpha)}(x, y)$ of (2.4) is recommended for $K=2$. For $K \geq 3$, (4.9) can be used. This is because one can choose $\alpha$ to fit in one's own application. To apply doubly adaptive biased coin designs, it is important to calculate their requisite sample sizes. Recently, $\mathrm{Hu}$ (2002) proposed some formulas to calculate sample sizes for response-adaptive randomization procedures with two treatments, $K=2$. These formulas can be used in doubly adaptive biased coin designs. How to calculate requisite sample sizes for $K \geq 3$ is a topic of future research.

\section{APPENDIX: PROOFS}

Let $\mathcal{F}_{m}=\sigma\left(\mathbf{X}_{1}, \ldots, \mathbf{X}_{m}, \xi_{1}, \ldots, \xi_{m}\right)$ be the sigma field generated by the previous $m$ stages. Then under $\mathcal{F}_{m-1} \mathbf{X}_{m}$ and $\xi_{m}$ are independent, and

$$
\mathrm{E}\left[\mathbf{X}_{m} \mid \mathcal{F}_{m-1}\right]=\mathbf{g}\left(\frac{\mathbf{N}_{m-1}}{m-1}, \widehat{\boldsymbol{\rho}}_{m-1}\right) .
$$

To prove Theorems 4.1 and 4.2, we first need some lemmas.

LEMMA A.1. Let $\mathbf{B}_{n, n}=\mathbf{I}$ and $\mathbf{B}_{n, i}=\prod_{j=i}^{n-1}\left(\mathbf{I}+j^{-1} \mathbf{H}\right)$. If two sequences of matrices $\mathbf{Q}_{n}$ and $\mathbf{P}_{n}$ satisfy

$$
\mathbf{Q}_{n}=\mathbf{P}_{n}+\sum_{k=1}^{n-1} \frac{\mathbf{Q}_{k}}{k} \mathbf{H}
$$

that is,

$$
\mathbf{Q}_{n}=\Delta \mathbf{P}_{n}+\mathbf{Q}_{n-1}\left(\mathbf{I}+\frac{\mathbf{H}}{n-1}\right), \quad n \geq 2
$$


where $\Delta \mathbf{P}_{1}=\mathbf{P}_{1}, \Delta \mathbf{P}_{n}=\mathbf{P}_{n}-\mathbf{P}_{n-1}, n \geq 2$, are the differences of $\mathbf{P}_{n}$, then

$$
\begin{aligned}
\mathbf{Q}_{n} & =\sum_{m=2}^{n} \Delta \mathbf{P}_{m} \mathbf{B}_{n, m}+\mathbf{Q}_{1} \mathbf{B}_{n, 1}=\left(\mathbf{Q}_{1}-\mathbf{P}_{1}\right) \mathbf{B}_{n, 1}+\sum_{m=1}^{n} \Delta \mathbf{P}_{m} \mathbf{B}_{n, m} \\
& =\left(\mathbf{Q}_{1}-\mathbf{P}_{1}\right) \mathbf{B}_{n, 1}+\mathbf{P}_{n}+\sum_{m=1}^{n-1} \mathbf{P}_{m} \frac{\mathbf{H}}{m} \mathbf{B}_{n, m+1} .
\end{aligned}
$$

Also,

(A.2) $\left\|\mathbf{B}_{n, m}\right\| \leq C(n / m)^{\lambda} \log ^{\nu-1}(n / m) \quad$ for all $m=1, \ldots, n, n \geq 1$, where, in the rest of the paper, $\log x=\ln (x \vee e)$.

PROOF. Equation (A.1) is easy by induction. Equation (A.2) follows because

$$
\begin{aligned}
\mathbf{T}^{-1} \mathbf{B}_{n, m} \mathbf{T} & =\prod_{j=m}^{n-1}\left(\mathbf{I}+j^{-1} \operatorname{diag}\left[0, \mathbf{J}_{2}, \ldots, \mathbf{J}_{s}\right]\right) \\
& =\operatorname{diag}\left[1, \prod_{j=m}^{n-1}\left(\mathbf{I}+j^{-1} \mathbf{J}_{2}\right), \ldots, \prod_{j=m}^{n-1}\left(\mathbf{I}+j^{-1} \mathbf{J}_{s}\right)\right]
\end{aligned}
$$

and $\left\|\prod_{j=m}^{n-1}\left(\mathbf{I}+j^{-1} \mathbf{J}_{t}\right)\right\| \leq C(n / m)^{\operatorname{Re}\left(\lambda_{t}\right)} \log ^{\nu_{t}-1}(n / m)$.

Lemma A.2. If two sequences of matrices $\mathbf{Q}_{n}$ and $\mathbf{P}_{n}$ satisfy

$$
\Delta \mathbf{Q}_{n}=\Delta \mathbf{P}_{n}+\frac{\mathbf{Q}_{n-1}}{n-1} \mathbf{H}+o\left(\frac{\left\|\mathbf{Q}_{n-1}\right\|}{n-1}\right)
$$

then, for any $\delta>0$,

$$
\mathbf{Q}_{n}=O(1) \sum_{m=1}^{n}\left\|\mathbf{P}_{m}\right\| \frac{1}{m}\left(\frac{1}{m}\right)^{\lambda+\delta}
$$

ProOF. Write

$$
\Delta \mathbf{Q}_{n}=\Delta \mathbf{P}_{n}+\frac{\mathbf{Q}_{n-1}}{n-1} \mathbf{H}+\frac{\left\|\mathbf{Q}_{n-1}\right\|}{n-1} \mathbf{A}_{n-1}, \quad n \geq 2,
$$

where $\left\|\mathbf{A}_{n}\right\| \rightarrow 0$. Then

$$
\Delta \mathbf{Q}_{n}=\left(\Delta \mathbf{P}_{n}+\frac{\left\|\mathbf{Q}_{n-1}\right\|}{n-1} \mathbf{A}_{n-1}\right)+\frac{\mathbf{Q}_{n-1}}{n-1} \mathbf{H} .
$$

From (A.1), it follows that

$$
\mathbf{Q}_{n}=\mathbf{P}_{n}+\sum_{m=1}^{n-1} \mathbf{P}_{m} \frac{\mathbf{H}}{m} \mathbf{B}_{n, m+1}+\sum_{m=2}^{n} \frac{\left\|\mathbf{Q}_{m-1}\right\|}{m-1} \mathbf{A}_{m-1} \mathbf{B}_{n, m}+\left(\mathbf{Q}_{1}-\mathbf{P}_{1}\right) \mathbf{B}_{n, 1} .
$$


Then by (A.2) there exists a constant $C_{0} \geq 1$ such that

(A.3) $\left\|\mathbf{Q}_{n}\right\| \leq C_{0} \sum_{m=1}^{n}\left\|\mathbf{P}_{m}\right\| \frac{1}{m}\left(\frac{n}{m}\right)^{\lambda+\delta / 2}+C_{0} \sum_{m=1}^{n-1} \frac{\left\|\mathbf{Q}_{m}\right\|}{m}\left\|\mathbf{A}_{m}\right\|\left(\frac{n}{m}\right)^{\lambda+\delta / 2}$.

Now we define a sequence of real numbers $D_{m} \geq 1$ such that

$$
\left\|\mathbf{Q}_{m}\right\| \leq C_{0} D_{m} \sum_{k=1}^{m}\left\|\mathbf{P}_{k}\right\| \frac{1}{k}\left(\frac{m}{k}\right)^{\lambda+\delta} .
$$

It is easy to define $D_{m}$ for $m=1, \ldots, 9$. Assume that $n \geq 10$ and $D_{m}$ is defined for $m=1, \ldots, n-1$. Let $n_{1}=[\sqrt{n}]$ and write

$$
\Gamma_{n}=\sum_{m=1}^{n}\left\|\mathbf{P}_{m}\right\| \frac{1}{m}\left(\frac{n}{m}\right)^{\lambda+\delta} .
$$

From (A.3), it follows that

$$
\begin{aligned}
\left\|\mathbf{Q}_{n}\right\| \leq & C_{0} \Gamma_{n}+C_{0} \sum_{m=1}^{n-1} C_{0} D_{m} \Gamma_{m}\left\|\mathbf{A}_{m}\right\| \frac{1}{m}\left(\frac{n}{m}\right)^{\lambda+\delta / 2} \\
\leq & C_{0} \Gamma_{n}+C_{0}^{2} \max _{m \leq n-1} D_{m} \sum_{k=1}^{n-1}\left\|\mathbf{P}_{k}\right\| \frac{1}{k}\left(\frac{n}{k}\right)^{\lambda+\delta} \sum_{m=k}^{n-1}\left\|\mathbf{A}_{m}\right\| \frac{n^{-\delta / 2}}{m^{1-\delta / 2}} \\
\leq & C_{0} \Gamma_{n}+C_{0}^{2} \max _{m \leq n-1} D_{m} \sum_{k=1}^{n}\left\|\mathbf{P}_{k}\right\| \frac{1}{k}\left(\frac{n}{k}\right)^{\lambda+\delta} \sum_{m=k}^{n_{1}}\left\|\mathbf{A}_{m}\right\| \frac{n^{-\delta / 2}}{m^{1-\delta / 2}} \\
& +C_{0}^{2} \max _{m \leq n-1} D_{m} \sum_{k=1}^{n}\left\|\mathbf{P}_{k}\right\| \frac{1}{k}\left(\frac{n}{k}\right)^{\lambda+\delta} \sum_{m=n_{1}}^{n-1}\left\|\mathbf{A}_{m}\right\| \frac{n^{-\delta / 2}}{m^{1-\delta / 2}} \\
\leq & C_{0}\left\{1+C_{0} \max _{m \leq n-1} D_{m} \frac{2}{\delta}\left(\max _{m \geq 1}\left\|\mathbf{A}_{m}\right\|\left(\frac{n_{1}+1}{n}\right)^{\delta / 2}+\max _{m \geq n_{1}}\left\|\mathbf{A}_{m}\right\|\right)\right\} \Gamma_{n},
\end{aligned}
$$

where $\sum_{m=k+1}^{k}(\cdot)=0$. Now define

$$
D_{n}=1+C_{0} \max _{m \leq n-1} D_{m} \frac{2}{\delta}\left(\max _{m \geq 1}\left\|\mathbf{A}_{m}\right\|\left(\frac{[\sqrt{n}]+1}{n}\right)^{\delta / 2}+\max _{m \geq[\sqrt{n}]}\left\|\mathbf{A}_{m}\right\|\right) .
$$

Next, it suffices to show the boundedness of $D_{n}$. Since $\left\|\mathbf{A}_{n}\right\| \rightarrow 0$, there exists an $n_{\delta}$ such that

$$
C_{0} \frac{2}{\delta}\left(\max _{m \geq 1}\left\|\mathbf{A}_{m}\right\|\left(\frac{[\sqrt{n}]+1}{n}\right)^{\delta / 2}+\max _{m \geq[\sqrt{n}]}\left\|\mathbf{A}_{m}\right\|\right) \leq \frac{1}{2} .
$$

Then

$$
D_{n} \leq 1+\frac{\max _{m \leq n-1} D_{m}}{2}, \quad n \geq n_{\delta},
$$


which, together with induction, implies that $D_{n} \leq 1+\max _{m \leq n_{\delta}-1} D_{m}$ for all $n \geq n_{\delta}$.

LemmA A.3. Let $\lambda_{0} \geq 0$ and $K_{0}>0$ be two real numbers and let $\left\{q_{n}\right\}$ be a sequence of nonnegative numbers and $\left\{p_{n}\right\}$ a sequence of real numbers for which

$$
q_{n} \leq\left(1+\frac{\lambda_{0}}{n-1}\right)\left(q_{n-1} \vee K_{0}\right)+\Delta p_{n}, \quad n \geq 2,
$$

where $\Delta p_{1}=p_{1}$ and $\Delta p_{n}=p_{n}-p_{n-1}, n \geq 2$. Then there exists a constant $C>0$ that depends only on $\lambda_{0}$ such that

$$
q_{n} \leq C\left\{\sum_{m=1}^{n} \frac{\left|p_{m}\right|}{m}\left(\frac{n}{m}\right)^{\lambda_{0}}+\max _{1 \leq m \leq n}\left(\frac{n}{m}\right)^{\lambda_{0}}\left|p_{m}\right|+\left(K_{0}+\left|q_{1}\right|\right) n^{\lambda_{0}}\right\} .
$$

PROOF. Without loss of generality, we assume that $K_{0}=1$. Let $b_{n, n}=1$,

$$
b_{n, m}=\prod_{i=m}^{n-1}\left(1+\frac{\lambda_{0}}{i}\right), \quad m=1,2, \ldots, n-1, n=1,2, \ldots,
$$

and $d_{n, m}=b_{n, m} \Delta p_{m}+b_{n, m-1} \Delta p_{m-1}+\cdots+b_{n, n} \Delta p_{n}, 1 \leq m \leq n$. Then

$$
\begin{aligned}
q_{n} \leq & \max \left\{b_{n, n-1} q_{n-1}+d_{n, n}, b_{n, n-1}+d_{n, n}\right\} \\
\leq & \max \left\{b_{n, n-1}\left[\left(1+\frac{\lambda_{0}}{n-1}\right)\left(q_{n-2} \vee 1\right)+\Delta p_{n-1}\right]\right. \\
& \left.+d_{n, n}, b_{n, n-1}+d_{n, n}\right\} \\
= & \max \left\{b_{n, n-2} q_{n-2}+b_{n, n-1} \Delta p_{n-1}+d_{n, n}, b_{n, n-2}\right. \\
& \left.\quad+b_{n, n-1} \Delta p_{n-1}+d_{n, n}, b_{n, n-1}+d_{n, n}\right\} \\
= & \max \left\{b_{n, n-2} q_{n-2}+d_{n, n-1}, b_{n, n-2}+d_{n, n-1}, b_{n, n-1}+d_{n, n}\right\} \\
\leq & \cdots \leq \max \left\{b_{n, n-m} q_{n-m}+d_{n, n-m+1}, b_{n, n-k}\right. \\
& \left.\quad+d_{n, n-k+1}: k=1, \ldots, m\right\} \\
\leq & \cdots \leq \max \left\{b_{n, 1} q_{1}+d_{n, 2}, b_{n, n-k}+d_{n, n-k+1}: k=1, \ldots, n-1\right\} \\
= & \max \left\{b_{n, 1} q_{1}+d_{n, 2}, b_{n, m}+d_{n, m+1}: m=1, \ldots, n\right\} .
\end{aligned}
$$

Note that

$$
\begin{aligned}
d_{n, m} & =\sum_{k=m}^{n} b_{n, k}\left(p_{k}-p_{k-1}\right)=\sum_{k=m}^{n-1}\left(b_{n, k}-b_{n, k+1}\right) p_{k}+b_{n, n} p_{n}-b_{n, m} p_{m-1} \\
& =\sum_{k=m}^{n-1} \lambda_{0} \frac{p_{k}}{k} b_{n, k+1}+p_{n}-b_{n, m} p_{m-1} .
\end{aligned}
$$


Also,

$$
\left|b_{n, m}\right| \leq C(n / m)^{\lambda_{0}}, \quad k=1, \ldots, n, n=1,2, \ldots
$$

It follows that

$$
\begin{aligned}
\left|d_{n, m}\right| & \leq C\left\{\sum_{k=m}^{n-1} \frac{\left|p_{k}\right|}{k}\left(\frac{n}{k}\right)^{\lambda_{0}}+\left|p_{n}\right|+\left(\frac{n}{m-1}\right)^{\lambda}\left|p_{m-1}\right|\right\} \\
& \leq C\left\{\sum_{k=1}^{n} \frac{\left|p_{k}\right|}{k}\left(\frac{n}{k}\right)^{\lambda_{0}}+\max _{1 \leq k \leq n}\left(\frac{n}{k}\right)^{\lambda}\left|p_{k}\right|\right\} .
\end{aligned}
$$

Now, by (A.4) we conclude that

$$
q_{n} \leq C\left\{\sum_{m=1}^{n} \frac{\left|p_{m}\right|}{m}\left(\frac{n}{m}\right)^{\lambda_{0}}+\max _{1 \leq m \leq n}\left(\frac{n}{m}\right)^{\lambda_{0}}\left|p_{m}\right|+\left(1+\left|q_{1}\right|\right) n^{\lambda_{0}}\right\}
$$

The lemma is proved.

LEMMA A.4. For each $k=1, \ldots, K$, we have

$$
\left\{N_{n, k} \rightarrow \infty\right\} \quad \text { a.s. implies }
$$

$$
\begin{cases}\widehat{\theta}_{n, k i} \rightarrow \theta_{k i}, & \text { if (A1), } \\ \widehat{\theta}_{n, k i}-\theta_{k i}=O\left(\sqrt{\frac{\log \log N_{n, k}}{N_{n, k}}}\right), & \text { if (A2), }\end{cases}
$$

$i=1, \ldots, d$.

Proof. For $k=1, \ldots, K$, define $\tau_{i}^{k}=\min \left\{j: N_{j, k}=i\right\}$, where $\min \{\varnothing\}=$ $+\infty$. Let $\left\{\boldsymbol{\eta}_{i, k}\right\}$ be an independent copy of $\left\{\boldsymbol{\xi}_{i, k}\right\}$, which is also independent of $\left\{\mathbf{X}_{i}\right\}$. Define $\boldsymbol{\Xi}_{i, k}=\boldsymbol{\xi}_{\tau_{i}^{k}, k} I\left\{\tau_{i}^{k}<+\infty\right\}+\eta_{i, k} I\left\{\tau_{i}^{k}=+\infty\right\}, i=1,2, \ldots$ Using the same argument of Doob (1936), we can show that $\left\{\boldsymbol{\Xi}_{m, k}, m=1,2, \ldots\right\}$ is a sequence of i.i.d. random vectors, with a common distribution the same as that of $\boldsymbol{\xi}_{1, k}$. Therefore, $n^{-1} \sum_{m=1}^{n} \boldsymbol{\Xi}_{m, k} \rightarrow \boldsymbol{\theta}_{k}$ a.s. if (A1), and $\sum_{m=1}^{n} \boldsymbol{\Xi}_{m, k}-n \boldsymbol{\theta}_{k}=$ $O\left((n \log \log n)^{1 / 2}\right)$ a.s. if (A2). Now, by noting that

$$
\widehat{\boldsymbol{\theta}}_{n, k}=\frac{1}{N_{n, k}+1}\left(\sum_{i=1}^{N_{n, k}} \boldsymbol{\Xi}_{m, k}+\boldsymbol{\theta}_{0, k}\right)
$$

on the event $\left\{N_{n, k} \rightarrow \infty\right\}$, (A.5) is proved.

LEMMA A.5. For the adaptive design 1, if conditions (A1), (B3) and (C1) are satisfied, then $N_{n, k} \rightarrow \infty$ a.s., $k=1, \ldots, K$, and

$$
\widehat{\boldsymbol{\Theta}}_{n} \rightarrow \boldsymbol{\Theta} \text { and } \widehat{\boldsymbol{\rho}}_{n} \rightarrow \mathbf{v} \quad \text { a.s. }
$$


Further, if condition (A2) is also satisfied, then

$$
\widehat{\theta}_{n, k i}-\theta_{k i}=O\left(\sqrt{\frac{\log \log N_{n, k}}{N_{n, k}}}\right) \quad \text { a.s., } k=1, \ldots, K, i=1, \ldots, d .
$$

Proof. By Lemma A.4 and the continuity of $\rho(\mathbf{y})$, it suffices to show that $N_{n, k} \rightarrow \infty$ a.s., $k=1, \ldots, K$. Note that, for each $k, k=1, \ldots, K$, on the event $\left\{\lim _{n \rightarrow \infty} N_{n, k}<\infty\right\}$ the sequence $\left\{\widehat{\theta}_{n, k i}\right\}$ takes a finite number of values. Also, on the event $\left\{\lim _{n \rightarrow \infty} N_{n, k}=\infty\right\} \widehat{\theta}_{n, k i} \rightarrow \theta_{k i}$ a.s. This shows that $\left\{\widehat{\boldsymbol{\Theta}}_{n}\right\}$ is almost surely a relatively compact set. Since $\rho(\mathbf{y}) \in(0,1)^{K}$ for any $\mathbf{y}$ on the closure of $\left\{\widehat{\boldsymbol{\Theta}}_{n}\right\}$, by the continuity of $\boldsymbol{\rho}(\mathbf{y})$ almost surely there exists a $0<\delta<1$ such that

$$
\widehat{\boldsymbol{\rho}}_{n}=\boldsymbol{\rho}\left(\widehat{\boldsymbol{\Theta}}_{n}\right) \in[\delta, 1)^{K}, \quad n=1,2, \ldots
$$

Note that

$$
\mathrm{P}\left(X_{n, k}=1 \mid \mathcal{F}_{n-1}\right)=g_{k}\left(\frac{\mathbf{N}_{n-1}}{n-1}, \widehat{\boldsymbol{\rho}}_{n-1}\right), \quad k=1, \ldots, K .
$$

For each $j=1, \ldots, K$, on the event $\left\{N_{n, j}=0, n=1,2, \ldots\right\}$, by (A.6) and condition (B2) we have

$$
\sum_{n=2}^{\infty} \mathrm{P}\left(X_{n, j}=1 \mid \mathcal{F}_{n-1}\right) \geq \sum_{n=2}^{\infty} c_{\delta}=+\infty \quad \text { a.s. },
$$

which implies $\left\{X_{n, j}=1\right.$, i.o. $\}=\left\{N_{n, j} \rightarrow \infty\right\}$ almost surely by the generalized Borel-Cantelli lemma [cf. Corollary 2.3 of Hall and Heyde (1980)]. This is a contradiction. Therefore,

$$
\lim _{n \rightarrow \infty} N_{n, j} \geq 1 \quad \text { a.s., } j=1, \ldots, K .
$$

Then, on the event $\left\{\lim _{n \rightarrow \infty} N_{n, k}<\infty\right\}$, by (A.6) and condition (B4) again,

$$
\begin{aligned}
\mathrm{P}\left(X_{n, k}=1 \mid \mathscr{F}_{n-1}\right) & \geq \frac{c_{\delta}}{2} \min \left\{\frac{N_{n-1, j}}{n-1}: j=1, \ldots, K\right\} \\
& \geq \frac{c_{\delta}}{2(n-1)} \quad \text { for } n \text { large enough a.s. }
\end{aligned}
$$

Then

$$
\sum_{n=2}^{\infty} \mathrm{P}\left(X_{n, k}=1 \mid \mathcal{F}_{n-1}\right)=+\infty \quad \text { a.s. }
$$

which implies $\left\{X_{n, k}=1\right.$, i.o. $\}=\left\{N_{n, k} \rightarrow \infty\right\}$ almost surely by the generalized Borel-Cantelli lemma again. This is also a contradiction. Therefore,

$$
N_{n, k} \rightarrow \infty \quad \text { a.s., } k=1, \ldots, K .
$$


Proof OF THEOREM 4.1. Recall that conditions (A1), (B1), (B2"), (B3) and (C1) are assumed. By Lemma A.5, $\widehat{\boldsymbol{\rho}}_{n} \rightarrow \mathbf{v}$ a.s. Let $\mathbf{M}_{n}=\sum_{m=1}^{n} \Delta \mathbf{M}_{m}$, where $\Delta \mathbf{M}_{m}=\mathbf{X}_{m}-\mathrm{E}\left[\mathbf{X}_{m} \mid \mathcal{F}_{m-1}\right]$. Then

$$
\begin{aligned}
\Delta\left(\mathbf{N}_{n}-n \mathbf{v}\right)= & \mathbf{X}_{n}-\mathbf{v}=\Delta \mathbf{M}_{n}+\mathbf{g}\left(\frac{\mathbf{N}_{n-1}}{n-1}, \widehat{\boldsymbol{\rho}}_{n-1}\right)-\mathbf{v} \\
= & \Delta \mathbf{M}_{n}+\left(\mathbf{g}\left(\frac{\mathbf{N}_{n-1}}{n-1}, \widehat{\boldsymbol{\rho}}_{n-1}\right)-\mathbf{g}\left(\frac{\mathbf{N}_{n-1}}{n-1}, \mathbf{v}\right)\right) \\
& +\mathbf{g}\left(\frac{\mathbf{N}_{n-1}}{n-1}, \mathbf{v}\right)-\mathbf{g}(\mathbf{v}, \mathbf{v}) .
\end{aligned}
$$

By condition (B1),

$$
\mathbf{g}\left(\frac{\mathbf{N}_{n-1}}{n-1}, \widehat{\boldsymbol{\rho}}_{n-1}\right)-\mathbf{g}\left(\frac{\mathbf{N}_{n-1}}{n-1}, \boldsymbol{v}\right) \rightarrow 0 \quad \text { a.s. }
$$

On the other hand, $\mathbf{M}_{n}=o(n)$ a.s. by the law of large numbers. We shall apply Lemma A.3. For each $k=1, \ldots, K$, let $q_{n}=q_{n}(k)=\left(\mathbf{N}_{n}-n \mathbf{v}\right) \mathbf{s}_{k}^{\prime}$ and

$$
\Delta p_{n}=\Delta p_{n}(k)=\Delta \mathbf{M}_{n} \mathbf{s}_{k}^{\prime}+\left(\mathbf{g}\left(\frac{\mathbf{N}_{n-1}}{n-1}, \widehat{\boldsymbol{\rho}}_{n-1}\right)-\mathbf{g}\left(\frac{\mathbf{N}_{n-1}}{n-1}, \mathbf{v}\right)\right) \mathbf{s}_{k}^{\prime}
$$

Then $p_{n}=\mathbf{M}_{n} \mathbf{s}_{k}^{\prime}+\sum_{m=1}^{n-1} o(1)=o(n)$ a.s. and

$$
q_{n}=\Delta p_{n}+q_{n-1}+\frac{\left(\mathbf{g}\left(\mathbf{N}_{n-1} /(n-1), \mathbf{v}\right)-\mathbf{g}(\mathbf{v}, \mathbf{v})\right) \mathbf{s}_{k}^{\prime}}{\left(\mathbf{N}_{n-1} /(n-1)-\mathbf{v}\right) \mathbf{s}_{k}^{\prime}} \frac{q_{n-1}}{n-1} .
$$

By condition $\left(\mathrm{B} 2^{\prime \prime}\right)$, if $q_{n-1} \geq 0$, then

$$
q_{n} \leq\left(1+\frac{\lambda_{0}}{n-1}\right) q_{n-1}+\Delta p_{n}
$$

On the other hand, if $q_{n-1} \leq 0$, then

$$
q_{n}=q_{n-1}+\left(\mathbf{X}_{n}-\mathbf{v}\right) \mathbf{s}_{k}^{\prime} \leq\left|\left(\mathbf{X}_{n}-\mathbf{v}\right) \mathbf{s}_{k}^{\prime}\right| \leq K_{1} .
$$

Also, $\left|\Delta p_{n}\right| \leq K_{2}$. If we choose $K_{0}=K_{1}+K_{2}$, then

$$
q_{n}^{+} \leq\left(1+\frac{\lambda_{0}}{n-1}\right)\left(q_{n-1}^{+} \vee K_{0}\right)+\Delta p_{n}
$$


So, from Lemma A.3 it follows that

$$
\begin{aligned}
q_{n}^{+} & \leq C\left\{\sum_{m=1}^{n} \frac{\left|p_{m}\right|}{m}\left(\frac{n}{m}\right)^{\lambda_{0}}+\max _{1 \leq m \leq n}\left(\frac{n}{m}\right)^{\lambda_{0}}\left|p_{m}\right|+\left(K_{0}+\left|q_{1}\right|\right) n^{\lambda_{0}}\right\} \\
& =\sum_{m=1}^{n} \frac{o(m)}{m}\left(\frac{n}{m}\right)^{\lambda_{0}}+\max _{1 \leq m \leq n}\left(\frac{n}{m}\right)^{\lambda_{0}} o(m)+O\left(n^{\lambda_{0}}\right)=o(n)
\end{aligned}
$$

since $0 \leq \lambda_{0}<1$. Thus,

$$
\limsup _{n \rightarrow \infty}\left(\frac{\mathbf{N}_{n}}{n}-\mathbf{v}\right) \mathbf{s}_{k}^{\prime} \leq 0 \quad \text { a.s., } k=1, \ldots, K .
$$

Now suppose that $\overline{\mathbf{v}}$ is a limit of one subsequence of $\left\{\mathbf{N}_{n} / n\right\}$. Then, by (A.7)

$$
(\overline{\mathbf{v}}-\mathbf{v}) \mathbf{s}_{k}^{\prime} \leq 0, \quad k=1, \ldots, K .
$$

All of the above $K$ inequalities must be equalities, since otherwise,

$$
0=(\overline{\mathbf{v}}-\mathbf{v}) \mathbf{1}^{\prime} c_{0}=(\overline{\mathbf{v}}-\mathbf{v}) \mathbf{S} 1^{\prime}=\sum_{k=1}^{K}(\overline{\mathbf{v}}-\mathbf{v}) \mathbf{s}_{k}^{\prime}<0,
$$

which is a contradiction. Therefore, $\overline{\mathbf{v}} \mathbf{s}_{k}^{\prime}=\mathbf{v} \mathbf{s}_{k}^{\prime}, k=1, \ldots, K$, that is, $\overline{\mathbf{v}} \mathbf{S}=\mathbf{v S}$. It follows that $\overline{\mathbf{v}}=\mathbf{v}$. Then $\mathbf{N}_{n} / n \rightarrow \mathbf{v}$ a.s. is proved. Theorem 4.1 is proved.

Proof OF THEOREM 4.2. Since $\mathbf{N}_{n} / n \rightarrow \mathbf{v}$ a.s. and $v_{k}>0, k=1, \ldots, K$, by Lemma A.4 we have

$$
\widehat{\boldsymbol{\Theta}}_{n}-\boldsymbol{\Theta}=O\left(\sqrt{\frac{\log \log n}{n}}\right) \quad \text { a.s. }
$$

Then, by condition (C2),

$$
\widehat{\boldsymbol{\rho}}_{n}-\mathbf{v}=\boldsymbol{\rho}\left(\widehat{\boldsymbol{\Theta}}_{n}\right)-\boldsymbol{\rho}(\boldsymbol{\Theta})=O\left(\sqrt{\frac{\log \log n}{n}}\right) \quad \text { a.s. }
$$

On the other hand, $\mathbf{M}_{n}=O(\sqrt{n \log \log n})$ by the law of the iterated logarithm. Therefore,

$$
\mathbf{M}_{n}+\sum_{m=1}^{n} O\left(\left\|\widehat{\boldsymbol{\rho}}_{n}-\mathbf{v}\right\|\right)=\mathbf{M}_{n}+\sum_{m=1}^{n} O\left(\sqrt{\frac{\log \log m}{m}}\right)=O(\sqrt{n \log \log n}) \quad \text { a.s. }
$$

Now, by condition (B4),

$$
\begin{aligned}
\Delta\left(\mathbf{N}_{n}-n \mathbf{v}\right) & =\mathbf{X}_{n}-\mathbf{v}=\Delta \mathbf{M}_{n}+\mathbf{g}\left(\frac{\mathbf{N}_{n-1}}{n-1}, \widehat{\boldsymbol{\rho}}_{n-1}\right)-\mathbf{g}(\mathbf{v}, \mathbf{v}) \\
& =\left(\frac{\mathbf{N}_{n-1}}{n-1}-\mathbf{v}\right) \mathbf{H}+o\left(\left\|\frac{\mathbf{N}_{n-1}}{n-1}-\mathbf{v}\right\|\right)+\Delta \mathbf{M}_{n}+O\left(\left\|\widehat{\boldsymbol{\rho}}_{n}-\mathbf{v}\right\|\right)
\end{aligned}
$$


Then, by Lemma A.2,

(A.9)

$$
\mathbf{N}_{n}-n \mathbf{v} \stackrel{\text { a.s. }}{=} \sum_{m=1}^{n} O(\sqrt{m \log \log m}) \frac{1}{m}\left(\frac{n}{m}\right)^{\lambda+\delta}
$$

$$
= \begin{cases}O(\sqrt{n \log \log n}), & \text { if } \lambda+\delta<1 / 2, \\ O\left(n^{\lambda+\delta}\right), & \text { if } \lambda+\delta>1 / 2 .\end{cases}
$$

The proof of Theorem 4.2 is completed by noting that $\delta>0$ can be chosen arbitrarily small.

Proof of Theorem 4.3. Let $\mathbf{Q}_{n}=\sum_{m=1}^{n} \Delta \mathbf{Q}_{m}$, where $\Delta \mathbf{Q}_{m}=\left(\Delta \mathbf{Q}_{m, 1}\right.$, $\left.\ldots, \Delta \mathbf{Q}_{m, K}\right)=\left(\Delta Q_{m, k i} ; i=1, \ldots, d, k=1, \ldots, K\right)$ and $\Delta Q_{m, k i}=X_{m, k}\left(\xi_{m, k i}-\right.$ $\left.\theta_{k i}\right) / v_{k}, i=1, \ldots, d, k=1, \ldots, K$. Then $\mathbf{Q}_{n}$ is a sequence of martingales in $\mathcal{R}^{K \times d}$, and $\mathbf{Q}_{n}=O(\sqrt{n \log \log n})$ a.s. by (A2) and the law of the iterated $\log$ arithm. By (A.9), it follows that

$$
\begin{aligned}
\widehat{\theta}_{n, k i}-\theta_{k i} & =\frac{1}{N_{n, k}+1}\left\{\sum_{m=1}^{n} X_{m, k} \xi_{m, k i}+\theta_{0, k i}\right\}-\theta_{k i} \\
& =\frac{v_{k}}{N_{n, k}+1}\left\{\sum_{m=1}^{n} X_{m, k} \frac{\xi_{m, k i}-\theta_{k i}}{v_{k}}\right\}+\frac{\theta_{0, k i}-\theta_{k i}}{N_{n, k}+1} \\
& =\frac{Q_{n, k i}}{n}+\left(\frac{v_{k}}{N_{n, k}+1}-\frac{1}{n}\right) Q_{n, k i}+\frac{\theta_{0, k i}-\theta_{k i}}{N_{n, k}+1} \\
& =\frac{Q_{n, k i}}{n}+O\left(\frac{\sqrt{n \log \log n}}{n^{2}}\right) O(\sqrt{n \log \log n})+O\left(\frac{1}{n}\right) \\
& =\frac{Q_{n, k i}}{n}+O\left(\frac{\log \log n}{n}\right) \quad \text { a.s., } k=1, \ldots, K, i=1, \ldots, d,
\end{aligned}
$$

that is,

$$
\widehat{\boldsymbol{\Theta}}_{n}-\boldsymbol{\Theta}=\frac{\mathbf{Q}_{n}}{n}+O\left(\frac{\log \log n}{n}\right)
$$

By (A.8) and condition (C2), we have

$$
\begin{aligned}
\widehat{\boldsymbol{\rho}}_{n}-\mathbf{v} & =\left.\left(\widehat{\boldsymbol{\Theta}}_{n}-\boldsymbol{\Theta}\right) \nabla(\boldsymbol{\rho})\right|_{\boldsymbol{\Theta}}+o\left(\left\|\widehat{\boldsymbol{\Theta}}_{n}-\boldsymbol{\Theta}\right\|^{1+\delta}\right) \\
& =\left.\frac{\mathbf{Q}_{n}}{n} \nabla(\boldsymbol{\rho})\right|_{\boldsymbol{\Theta}}+o\left(n^{-1 / 2-\delta / 3}\right) \quad \text { a.s. }
\end{aligned}
$$


On the other hand, by (3.3) and (A.8)-(A.10) we have

$$
\begin{aligned}
\Delta\left(\mathbf{N}_{n}-\right. & n \mathbf{v})=\mathbf{X}_{n}-\mathbf{v}=\Delta \mathbf{M}_{n}+\mathbf{g}\left(\frac{\mathbf{N}_{n-1}}{n-1}, \widehat{\boldsymbol{\rho}}_{n-1}\right)-\mathbf{g}(\mathbf{v}, \mathbf{v}) \\
= & \Delta \mathbf{M}_{n}+\mathbf{f}\left(\frac{\mathbf{N}_{n-1}}{n-1}, \widehat{\boldsymbol{\Theta}}_{n-1}\right)-\mathbf{g}(\mathbf{v}, \mathbf{v}) \\
= & \Delta \mathbf{M}_{n}+\left(\frac{\mathbf{N}_{n-1}}{n-1}-\mathbf{v}\right) \mathbf{H}+\left.\left(\widehat{\boldsymbol{\Theta}}_{n-1}-\boldsymbol{\Theta}\right) \nabla(\boldsymbol{\rho})\right|_{\boldsymbol{\Theta}} \mathbf{E} \\
& +o\left(\left\|\frac{\mathbf{N}_{n-1}}{n-1}-\mathbf{v}\right\|^{1+\delta}\right)+o\left(\left\|\widehat{\boldsymbol{\Theta}}_{n-1}-\boldsymbol{\Theta}\right\|^{1+\delta}\right) \\
= & \Delta \mathbf{M}_{n}+\left.\frac{\mathbf{Q}_{n-1}}{n-1} \nabla(\rho)\right|_{\Theta} \mathbf{E} \\
& +\left(\frac{\mathbf{N}_{n-1}-(n-1) \mathbf{v}}{n-1}\right) \mathbf{H}+o(n-1 / 2-\delta / 3) \\
= & \Delta \mathbf{M}_{n}+\left.\frac{\mathbf{Q}_{n}}{n} \nabla(\rho)\right|_{\Theta} \mathbf{E}+\left(\frac{\mathbf{N}_{n-1}-(n-1) \mathbf{v}}{n-1}\right) \mathbf{H}+o\left(n^{-1 / 2-\delta / 3}\right)
\end{aligned}
$$

Then, by Lemma A.1, it follows that

$$
\begin{aligned}
\mathbf{N}_{n}-n \mathbf{v}= & \sum_{m=1}^{n}\left(\Delta \mathbf{M}_{m}+\left.\frac{\mathbf{Q}_{m}}{m} \nabla(\rho)\right|_{\Theta} \mathbf{E}+o\left(m^{-1 / 2-\delta / 3}\right)\right) \mathbf{B}_{n, m} \quad \text { a.s. } \\
= & \sum_{m=1}^{n} \Delta \mathbf{M}_{m} \mathbf{B}_{n, m}+\left.\sum_{m=1}^{n} \sum_{k=1}^{m} \Delta \mathbf{Q}_{k} \nabla(\boldsymbol{\rho})\right|_{\Theta} \mathbf{E} \frac{1}{m} \mathbf{B}_{n, m} \\
& +\sum_{m=1}^{n} o\left(m^{-1 / 2-\delta / 3}\right)\left(\frac{n}{m}\right)^{\lambda} \log ^{\nu-1}\left(\frac{n}{m}\right) \\
\text { A.12) } & \sum_{m=1}^{n} \Delta \mathbf{M}_{m} \mathbf{B}_{n, m}+\left.\sum_{m=1}^{n} \Delta \mathbf{Q}_{m} \nabla(\boldsymbol{\rho})\right|_{\Theta} \mathbf{E} \sum_{k=m}^{n} \frac{1}{k} \mathbf{B}_{n, k}+o\left(n^{-1 / 2-\delta / 3}\right) \\
:= & \mathbf{U}_{n}+o\left(n^{-1 / 2-\delta / 3}\right) \quad \text { a.s. }
\end{aligned}
$$

Note that $\mathbf{U}_{n}$ is a sum of martingale differences, and for some $0<\varepsilon<1 / \lambda-2$,

$$
\begin{aligned}
& \frac{1}{n^{1+\varepsilon / 2}} \sum_{m=1}^{n}\left\{\left\|\Delta \mathbf{M}_{m} \mathbf{B}_{n, m}\right\|^{2+\varepsilon}+\left\|\Delta \mathbf{Q}_{m}\right\|^{2+\varepsilon}+\left\|\left.\Delta \mathbf{Q}_{m} \nabla(\boldsymbol{\rho})\right|_{\Theta} \mathbf{E} \sum_{k=m}^{n} \frac{1}{k} \mathbf{B}_{n, k}\right\|^{2+\varepsilon}\right\} \\
& \leq \frac{C}{n^{1+\varepsilon / 2}} \sum_{m=1}^{n}\left\{\left(\left(\frac{n}{m}\right)^{\lambda} \log ^{\nu-1}\left(\frac{n}{m}\right)\right)^{2+\varepsilon}+\left(\sum_{k=m}^{n} \frac{1}{k}\left(\frac{n}{k}\right)^{\lambda} \log ^{\nu-1}\left(\frac{n}{k}\right)\right)^{2+\varepsilon}\right\} \\
& \leq \frac{C}{n^{1+\varepsilon / 2}} \sum_{m=1}^{n}\left\{\left(\frac{n}{m}\right)^{\lambda} \log ^{\nu-1}\left(\frac{n}{m}\right)\right\}^{2+\varepsilon} \leq \frac{C}{n^{\varepsilon / 2}} \rightarrow 0 ;
\end{aligned}
$$


that is, the Lindberg condition is satisfied. On the other hand, it is easily seen that

$$
\begin{aligned}
& \operatorname{Var}\left[\Delta \mathbf{M}_{n} \mid \mathcal{F}_{n-1}\right] \\
& =\mathrm{E}\left[\operatorname{diag}\left(\mathbf{X}_{n}\right) \mid \mathcal{F}_{n-1}\right]-\left(\mathrm{E}\left[\mathbf{X}_{n} \mid \mathcal{F}_{n-1}\right]\right)^{\prime} \mathrm{E}\left[\mathbf{X}_{n} \mid \mathcal{F}_{n-1}\right] \\
& =\operatorname{diag}\left\{g\left(\frac{\mathbf{N}_{n-1}}{n-1}, \widehat{\boldsymbol{\rho}}_{n-1}\right)\right\}-\left\{g\left(\frac{\mathbf{N}_{n-1}}{n-1}, \widehat{\boldsymbol{\rho}}_{n-1}\right)\right\}^{\prime} g\left(\frac{\mathbf{N}_{n-1}}{n-1}, \widehat{\boldsymbol{\rho}}_{n-1}\right) \\
& \rightarrow \operatorname{diag}\{g(\mathbf{v}, \mathbf{v})\}-\{g(\mathbf{v}, \mathbf{v})\}^{\prime} g(\mathbf{v}, \mathbf{v}) \\
& =\operatorname{diag}(\mathbf{v})-\mathbf{v}^{\prime} \mathbf{v}=\Sigma_{1} \quad \text { a.s. } \\
& \operatorname{Cov}\left\{\Delta \mathbf{M}_{n}, \Delta \mathbf{Q}_{n} \mid \mathcal{F}_{n-1}\right\}=\mathbf{0} \quad \text { a.s. }
\end{aligned}
$$

Also,

$$
\begin{aligned}
\operatorname{Cov}[ & \left.\Delta Q_{n, k i}, \Delta Q_{n, l j} \mid \mathcal{F}_{n-1}\right] \\
& =\operatorname{Cov}\left[\frac{X_{n, k}\left(\xi_{n, k i}-\theta_{k i}\right)}{v_{k}}, \frac{X_{n, l}\left(\xi_{n, l j}-\theta_{l j}\right)}{v_{l}} \mid \mathcal{F}_{n-1}\right]=0, \quad k \neq l,
\end{aligned}
$$

and

$$
\begin{aligned}
\operatorname{Cov}[ & \left.\Delta Q_{n, k i}, \Delta Q_{n, k j} \mid \mathcal{F}_{n-1}\right] \\
& =\frac{1}{v_{k}^{2}} g_{k}\left(\frac{N_{n-1,1}}{n-1}, \widehat{\boldsymbol{\rho}}_{n-1, k}\right) \operatorname{Cov}\left\{\xi_{1, k i}, \xi_{1, k j}\right\} \\
& \rightarrow \frac{1}{v_{k}} \operatorname{Cov}\left\{\xi_{1, k i}, \xi_{1, k j}\right\} \quad \text { a.s., }
\end{aligned}
$$

that is,

$$
\operatorname{Var}\left[\Delta \mathbf{Q}_{n, k} \mid \mathcal{F}_{n-1}\right] \rightarrow \frac{1}{v_{k}} \mathbf{V}_{k}, \quad k=1, \ldots, K
$$

and

$$
\operatorname{Var}\left[\Delta \mathbf{Q}_{n} \mid \mathcal{F}_{n-1}\right] \rightarrow \mathbf{V} \quad \text { a.s. }
$$

Since $\mathrm{E}\left\|\Delta \mathbf{M}_{n}\right\|^{2+\varepsilon}+\mathrm{E}\left\|\Delta \mathbf{Q}_{n}\right\|^{2+\varepsilon} \leq C, n \geq 1$, the above conditioned covariances are also convergent in $L_{1}$. It follows that, for any $0<s<t<1$,

$$
\begin{aligned}
\operatorname{Cov}\left(\mathbf{U}_{[n s]}, \mathbf{U}_{[n t]}\right) & \\
= & \sum_{m=1}^{[n s]} \mathbf{B}_{[n s], m}^{\prime}\left(\boldsymbol{\Sigma}_{1}+o(1)\right) \mathbf{B}_{[n t], m} \\
& +\sum_{m=1}^{[n s]}\left(\sum_{k=m}^{[n s]} \frac{1}{k} \mathbf{B}_{[n s], k}\right)^{\prime}\left(\boldsymbol{\Sigma}_{2}+o(1)\right)\left(\sum_{k=m}^{[n t]} \frac{1}{k} \mathbf{B}_{[n t], k}\right)
\end{aligned}
$$




$$
\begin{aligned}
= & \int_{1}^{[n s]}\left(\frac{[n s]}{x}\right)^{\mathbf{H}^{\prime}} \boldsymbol{\Sigma}_{1}\left(\frac{[n t]}{x}\right)^{\mathbf{H}} d x \\
& +\int_{1}^{[n s]} d x\left[\int_{x}^{[n s]} \frac{1}{y}\left(\frac{[n s]}{y}\right)^{\mathbf{H}} d y\right]^{\prime} \boldsymbol{\Sigma}_{2}\left[\int_{x}^{[n t]} \frac{1}{y}\left(\frac{[n t]}{y}\right)^{\mathbf{H}} d y\right] \\
& +o(1) \sum_{m=1}^{n}\left(\frac{n}{m}\right)^{2 \lambda} \log ^{2 v-2}\left(\frac{n}{m}\right) \\
= & n \int_{1 / n}^{s}\left(\frac{s}{x}\right)^{\mathbf{H}^{\prime}} \boldsymbol{\Sigma}_{1}\left(\frac{t}{x}\right)^{\mathbf{H}} d x \\
& +n \int_{1 / n}^{s} d x\left[\int_{x}^{s} \frac{1}{y}\left(\frac{s}{y}\right)^{\mathbf{H}} d y\right]^{\prime} \boldsymbol{\Sigma}_{2}\left[\int_{x}^{t} \frac{1}{y}\left(\frac{t}{y}\right)^{\mathbf{H}} d y\right]+o(n) \\
= & n \int_{0}^{s}\left(\frac{s}{x}\right)^{\mathbf{H}^{\prime}} \boldsymbol{\Sigma}_{1}\left(\frac{t}{x}\right)^{\mathbf{H}} d x \\
& +n \int_{0}^{s} d x\left[\int_{x}^{s} \frac{1}{y}\left(\frac{s}{y}\right)^{\mathbf{H}} d y\right]^{\prime} \boldsymbol{\Sigma}_{2}\left[\int_{x}^{t} \frac{1}{y}\left(\frac{t}{y}\right)^{\mathbf{H}} d y\right]+o(n) \\
= & n \boldsymbol{\Lambda}_{11}(s, t)+o(n),
\end{aligned}
$$

and

(A.14)

$$
\begin{aligned}
\operatorname{Cov}\left[\left.\mathbf{Q}_{[n s]} \nabla(\boldsymbol{\rho})\right|_{\boldsymbol{\Theta}}, \mathbf{U}_{[n t]}\right] \\
=\left(\boldsymbol{\Sigma}_{3} \mathbf{E}+o(1)\right) \sum_{m=1}^{[n s]}\left(\sum_{k=m}^{[n t]} \frac{1}{k} \mathbf{B}_{[n t], k}\right) \\
=n \boldsymbol{\Sigma}_{3} \mathbf{E} \int_{1 / n}^{s} d x\left[\int_{x}^{t} \frac{t}{y}\left(\frac{t}{y}\right)^{\mathbf{H}} d y\right]+o(n) \\
=n \boldsymbol{\Sigma}_{3} \mathbf{E} \int_{0}^{s} d x\left[\int_{x}^{t} \frac{t}{y}\left(\frac{t}{y}\right)^{\mathbf{H}} d y\right]+o(n) \\
=n \mathbf{\Lambda}_{21}(s, t)+o(n),
\end{aligned}
$$

$\operatorname{Cov}\left[\left.\mathbf{Q}_{[n t]} \nabla(\boldsymbol{\rho})\right|_{\boldsymbol{\Theta}}, \mathbf{U}_{[n s]}\right]$

$$
\begin{aligned}
& =\sum_{m=1}^{[n s]}\left(\sum_{k=m}^{[n s]} \frac{1}{k} \mathbf{B}_{[n s], k}\right)^{\prime}\left(\mathbf{E}^{\prime} \boldsymbol{\Sigma}_{3}+o(1)\right) \\
& =n \int_{1 / n}^{s} d x\left[\int_{x}^{s} \frac{s}{y}\left(\frac{s}{y}\right)^{\mathbf{H}} d y\right]^{\prime} \mathbf{E}^{\prime} \boldsymbol{\Sigma}_{3}+o(n) \\
& =n \int_{0}^{s} d x\left[\int_{x}^{s} \frac{s}{y}\left(\frac{s}{y}\right)^{\mathbf{H}} d y\right] \mathbf{E}^{\prime} \boldsymbol{\Sigma}_{3}+o(n) \\
& =n s \boldsymbol{\Lambda}_{12}+o(n)
\end{aligned}
$$


and

$$
\begin{aligned}
\operatorname{Cov}\left[\left.\mathbf{Q}_{[n s]} \nabla(\boldsymbol{\rho})\right|_{\boldsymbol{\Theta}},\left.\mathbf{Q}_{[n t]} \nabla(\boldsymbol{\rho})\right|_{\boldsymbol{\Theta}}\right] \\
\quad=n s\left(\boldsymbol{\Sigma}_{3}+o(1)\right)=n s \boldsymbol{\Sigma}_{3}+o(n),
\end{aligned}
$$

where

$$
\begin{aligned}
\boldsymbol{\Lambda}_{11}(s, t)= & \int_{0}^{s}\left(\frac{s}{x}\right)^{\mathbf{H}^{\prime}} \boldsymbol{\Sigma}_{1}\left(\frac{t}{x}\right)^{\mathbf{H}} d x \\
& +\int_{0}^{s} d x\left[\int_{x}^{s} \frac{1}{y}\left(\frac{s}{y}\right)^{\mathbf{H}} d y\right]^{\prime} \boldsymbol{\Sigma}_{2}\left[\int_{x}^{t} \frac{1}{y}\left(\frac{t}{y}\right)^{\mathbf{H}} d y\right], \\
\boldsymbol{\Lambda}_{21}(s, t)= & \boldsymbol{\Sigma}_{3} \mathbf{E} \int_{0}^{s} d x\left[\int_{x}^{t} \frac{t}{y}\left(\frac{t}{y}\right)^{\mathbf{H}} d y\right] .
\end{aligned}
$$

This shows that the limiting covariance function of $n^{-1 / 2}\left(\mathbf{U}_{[n t]},\left.\mathbf{Q}_{[n t]} \nabla(\boldsymbol{\rho})\right|_{\Theta}\right)$ agrees with the covariance function of $\left(\mathbf{G}_{t}, \mathbf{B}_{t} \Sigma_{3}^{1 / 2}\right)$. So by the weak convergence of the martingale [cf. Theorem 4.1 of Hall and Heyde (1980)],

$$
n^{-1 / 2}\left(\mathbf{U}_{[n t]},\left.\mathbf{Q}_{[n t]} \nabla(\rho)\right|_{\Theta}\right) \stackrel{D}{\rightarrow}\left(\mathbf{G}_{t}, \mathbf{B}_{t} \Sigma_{3}^{1 / 2}\right) .
$$

The proof of Theorem 4.3 is now complete by noting (A.11) and (A.12).

Acknowledgments. Part of Feifang Hu's research was done while visiting the Department of Biometrics, Cornell University. He thanks the department for its hospitality and support. Special thanks go to anonymous referees and an associate editor for the constructive comments, which led to a much improved version of the paper.

\section{REFERENCES}

BAI, Z. D. and HU, F. (1999). Asymptotic theorems for urn models with nonhomogeneous generating matrices. Stochastic Process. Appl. 80 87-101.

BAi, Z. D., Hu, F. and Rosenberger, W. F. (2002). Asymptotic properties of adaptive designs for clinical trials with delayed response. Ann. Statist. 30 122-139.

Doob, J. L. (1936). Note on probability. Ann. Math. 37 363-367.

EFron, B. (1971). Forcing a sequential experiment to be balanced. Biometrika 58 403-417.

EISELE, J. (1994). The doubly adaptive biased coin design for sequential clinical trials. J. Statist. Plann. Inference 38 249-262.

EISElE, J. (1995). Biased coin designs: Some properties and applications. In Adaptive Designs (N. Flournoy and W. F. Rosenberger, eds.) 48-64. IMS, Hayward, CA.

EISEle, J. and Woodroofe, M. (1995). Central limit theorems for doubly adaptive biased coin designs. Ann. Statist. 23 234-254. 
Flournoy, N. and Rosenberger, W. F., eds. (1995). Adaptive Designs. IMS, Hayward, CA.

HALl, P. and Heyde, C. C. (1980). Martingale Limit Theory and Its Applications. Academic Press, London.

HAYre, L. S. (1979). Two-population sequential tests with three hypotheses. Biometrika 66 465-474.

HU, F. (2002). Sample size for response-adaptive randomization procedures. Unpublished manuscript.

Hu, F. and Rosenberger, W. F. (2003). Optimality, variability, power: Evaluating responseadaptive randomization procedures for treatment comparisons. J. Amer. Statist. Assoc. To appear.

IVAnova, A. and Rosenberger, W. F. (2001). Adaptive designs for clinical trials with highly successful treatments. Drug Information J. 35 1087-1093.

Jennison, C. and Turnbull, B. W. (2000). Group Sequential Methods with Applications to Clinical Trials. Chapman and Hall/CRC Press, Boca Raton, FL.

Matthews, P. C. and Rosenberger, W. F. (1997). Variance in randomized play-the-winner clinical trials. Statist. Probab. Lett. 35 223-240.

Melfi, V. and PAGE, C. (1998). Variability in adaptive designs for estimation of success probabilities. In New Developments and Applications in Experimental Design (N. Flournoy, W. F. Rosenberger and W. K. Wong, eds.) 106-114. IMS, Hayward, CA.

Melfi, V. and Page, C. (2000). Estimation after adaptive allocation. J. Statist. Plann. Inference 87 353-363.

Melfi, V., Page, C. and Geraldes, M. (2001). An adaptive randomized design with application to estimation. Canad. J. Statist. 29 107-116.

Robbins, H. (1952). Some aspects of the sequential design of experiments. Bull. Amer. Math. Soc. $58527-535$.

Rosenberger, W. F. (1996). New directions in adaptive designs. Statist. Sci. 11 137-149.

Rosenberger, W. F. (2002). Randomized urn models and sequential design (with discussion). Sequential Anal. 21 1-41.

Rosenberger, W. F. and Lachin, J. M. (2002). Randomization in Clinical Trials. Wiley, New York.

Rosenberger, W. F., Stallard, N., Ivanova, A., Harper, C. and Ricks, M. (2001). Optimal adaptive designs for binary response trials. Biometrics 57 909-913.

Smith, R. L. (1984). Properties of biased coin designs in sequential clinical trials. Ann. Statist. 12 1018-1034.

Smythe, R. T. (1996). Central limit theorems for urn models. Stochastic Process. Appl. 65 115-137.

Smythe, R. T. and Rosenberger, W. F. (1995). Play-the-winner designs, generalized Pólya urns, and Markov branching processes. In Adaptive Designs (N. Flournoy and W. F. Rosenberger, eds.) 13-22. IMS, Hayward, CA.

Thompson, W. R. (1933). On the likelihood that one unknown probability exceeds another in view of the evidence of the two samples. Biometrika 25 285-294.

WEI, L. J. (1978). The adaptive biased coin design for sequential experiments. Ann. Statist. 6 92-100.

WeI, L. J. (1979). The generalized Pólya's urn design for sequential medical trials. Ann. Statist. 7 291-296.

WEI, L. J. (1988). Exact two-sample permutation tests based on the randomized play-the-winner rule. Biometrika 75 603-606.

WeI, L. J. and DURHAM, S. (1978). The randomized pay-the-winner rule in medical trials. J. Amer. Statist. Assoc. 73 840-843. 
Wei, L. J., Smythe, R. T. and Smith, R. L. (1986). $K$-treatment comparisons with restricted randomization rules in clinical trials. Ann. Statist. 14 265-274.

Zelen, M. (1969). Play the winner rule and the controlled clinical trial. J. Amer. Statist. Assoc. 64 131-146.

DEPARTMENT OF STATISTICS

HALSEY HALL

UNIVERSITY OF VIRGINIA

CHARLOTTES VILLE, ViRginia 22904-4135

USA

E-MAIL: fh6e@virginia.edu
DEPARTMENT OF MATHEMATICS ZHEJIANG UNIVERSITY

XIXI CAMPUS

ZHEJIANG, HANGZHOU 310028

PEOPLE'S REPUBLIC OF CHINA

E-MAIL: 1xzhang@mail.hz.zj.cn 